\title{
Warps, grids and curvature in triple vector bundles
}

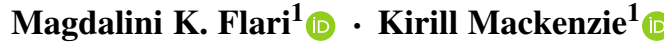

Received: 26 March 2018 / Revised: 14 May 2018 / Accepted: 22 May 2018 /

Published online: 8 June 2018

(C) The Author(s) 2018

\begin{abstract}
A triple vector bundle is a cube of vector bundle structures which commute in the (strict) categorical sense. A grid in a triple vector bundle is a collection of sections of each bundle structure with certain linearity properties. A grid provides two routes around each face of the triple vector bundle, and six routes from the base manifold to the total manifold; the warps measure the lack of commutativity of these routes. In this paper we first prove that the sum of the warps in a triple vector bundle is zero. The proof we give is intrinsic and, we believe, clearer than the proof using decompositions given earlier by one of us. We apply this result to the triple tangent bundle $T^{3} M$ of a manifold and deduce (as earlier) the Jacobi identity. We further apply the result to the triple vector bundle $T^{2} A$ for a vector bundle $A$ using a connection in $A$ to define a grid in $T^{2} A$. In this case the curvature emerges from the warp theorem.
\end{abstract}

Keywords Triple vector bundles · Double vector bundles · Connections · Curvature

Mathematics Subject Classification 53C05 - 18D05 - 18D35 - 55R65

凶 Magdalini K. Flari

mkflari1@sheffield.ac.uk

Kirill Mackenzie

K.Mackenzie@sheffield.ac.uk

http://kchmackenzie.staff.shef.ac.uk/

1 School of Mathematics and Statistics, University of Sheffield, Sheffield S3 7RH, UK 


\section{Introduction}

\subsection{Double vector bundles, grids and warps}

Double vector bundles arise naturally in Poisson geometry, in the connection theory of vector bundles, and generally in the study of geometric objects with two compatible structures. Double vector bundles have been "floating around" since at least Dieudonné's [4] treatment of connection theory, but the first systematic and general treatment was provided by Pradines [21]. A recent account with references is [16, Chap. 9]. We briefly recall the necessary facts.

A double vector bundle consists first of all of a square of vector bundles as shown in the first figure of (1). There are two vector bundle structures on $D$, with bases $A$ and $B$, each of which is itself a vector bundle on base $M$; the two structures on $D$ commute in the categorical sense (see below), and the map $D \rightarrow A \times{ }_{M} B$ formed by the two bundle projections is a surjective submersion. We call this the double source map and denote it by $\downarrow$; that $\downarrow$ is a surjective submersion was proved by Li-Bland and Ševera [13]. ${ }^{1}$ We further assume that $\downarrow$ has a right-inverse $\Sigma: A \times_{M} B \rightarrow D$ that is linear in both $A$ and $B$; we call this a sigma-map.
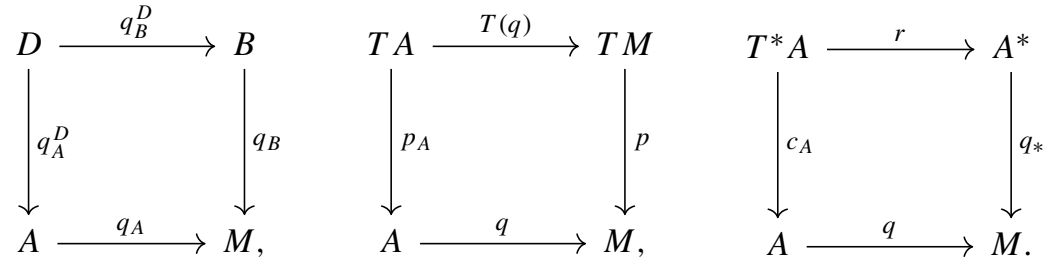

The second and third figures in (1) show two standard examples arising from an arbitrary vector bundle $A$. If $A$ has a Poisson structure, then it is linear if and only if the associated map $T^{*} A \rightarrow T A$ is a morphism of double vector bundles (in an obvious sense) of the structures above. When this is so, $A$ is the dual of a Lie algebroid [3]. The third structure was introduced in global form in [19]. We give more details on this double vector bundle in Example 2. Double vector bundles also arise in the Lie theory of double Lie groupoids [17]; we will not consider this theory here.

Each element $d$ of a double vector bundle $D$ may be represented in outline by the diagram in (2) which shows the projections of $d$ under the two bundle projections. Given another element $d^{\prime}$ as shown, the sum over $A$ has the outline shown in the third figure.
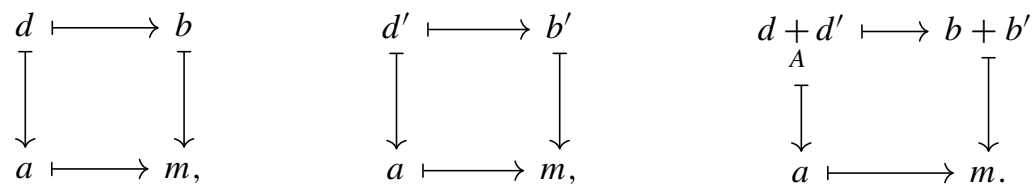

\footnotetext{
1 We are grateful to Rajan Mehta for pointing this reference out to us.
} 
The statement that the two vector bundle structures on $D$ 'commute in the categorical sense' implies for the additions that

$$
\left(d_{1}+\underset{A}{+} d_{2}\right)+\underset{B}{+}\left(d_{3}+d_{4}\right)=\left(d_{1}+d_{B}\right) \underset{A}{+}\left(d_{2}+d_{B}\right),
$$

where $\left(d_{i} ; a_{i}, b_{i} ; m\right), i=1, \ldots, 4$, have $a_{1}=a_{2}, a_{3}=a_{4}, b_{1}=b_{3}$ and $b_{2}=b_{4}$. Equation (3) is the key interchange law for double vector bundles. There are similar conditions involving the scalar multiplications, see [16, §9.1].

It follows that for elements $d$ which project to zeros under both bundle projections, the two additions, and the scalar multiplications, coincide. Under these operations the set of such elements forms a vector bundle over $M$, called the core of $D$ [21], usually denoted by $C$.

The core $C$ is a submanifold of $D$; every element of $C$ is an element of $D$. When working with examples, the core can usually be identified with a familiar vector bundle and it can be important to distinguish between elements of this bundle and the corresponding element of the double vector bundle. For example, the core of the double vector bundle $T A$, the middle diagram in (1), can be identified with $A$ itself, [16, 9.1.7]. Therefore, an element $a$ of the core $A$, can be viewed either as an element of $A$, or as an element of $T A$. In the latter case, we denote it by $\bar{a} \in T A$. For general double vector bundles and triple vector bundles, this distinction is usually not necessary, so in Sects. 2, 3, and 4, we will not write bars over core elements. This distinction will be made clearly in Sect. 5 .

Now suppose that $(d ; a, b ; m)$ and $\left(d^{\prime} ; a^{\prime}, b^{\prime} ; m\right)$ have $a=a^{\prime}$ and $b=b^{\prime}$. Then there is a unique $c \in C$ such that

$$
d=d_{A}^{\prime}+\underset{B}{+}\left(c+\tilde{0}_{a}\right)=d^{\prime}+\underset{A}{+}\left(c+\tilde{0}_{b}\right) .
$$

In equations of this type, what is important is that $d-d^{\prime}$, calculated in either vector bundle structure of the double vector bundle $D$, results in the same core element $c$ plus an appropriate zero. In Sect. 2.2, and particularly in Sects. 5 and 6, we will write such equations succinctly as $d-d^{\prime} \triangleright c$. Expressions of this form are useful in summarizing detailed calculations. We find, however, that the $\triangleright$ notation is not practical for detailed work; we have not attempted to develop a calculus for working with $\triangleright$.

We now describe the original motivating example for the concepts of grid and warp.

In the 1988 edition of their book [1, p. 297], Abraham, Marsden and Raţiu gave the following formula for the Lie bracket of vector fields $X$ and $Y$ on a manifold $M$,

$$
T(Y)(X(m))-\widetilde{X}(Y(m))=([X, Y](m))^{\uparrow}(Y(m))
$$

where $\tilde{X}$ is the complete lift of $X$ to a vector field on $T M$ and the uparrow denotes the vertical lift to $T_{Y(m)} T M$ of the vector $[X, Y](m)$. The complete lift, or tangent lift, $\widetilde{X}$ is $J \circ T(X)$ where $J: T^{2} M \rightarrow T^{2} M$ is the canonical involution which interchanges the two bundle structures on $T^{2} M$. The double vector bundle $T^{2} M$ is a special case of the middle diagram of (1), where $A=T M$, and its core vector bundle is yet a third 
copy of $T M$. The left-hand side of (5) is encapsulated in (6).

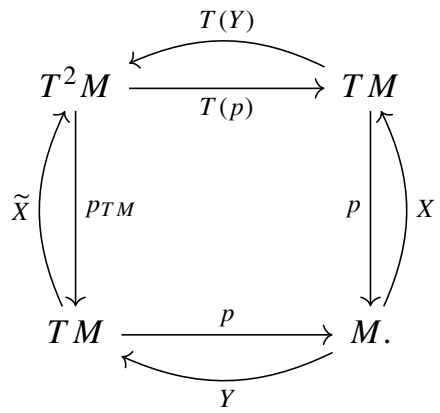

If we look at the elements $T(Y)(X(m))$ and $\tilde{X}(Y(m))$, we see that they have the same outlines
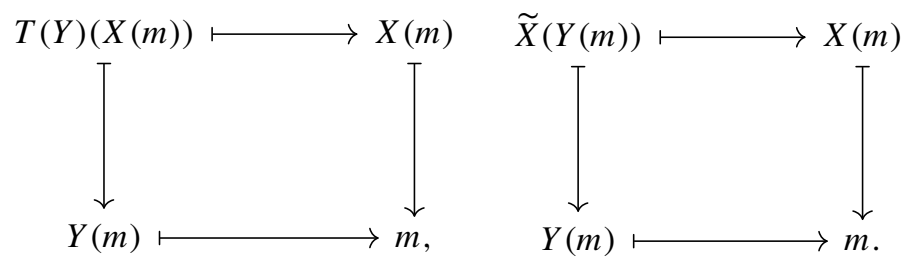

The two elements determine a unique core element $c \in T M$. Taking $d=$ $T(Y)(X(m))$ and $d^{\prime}=\widetilde{X}(Y(m))$ in $(4)$, we have

$$
T(Y)(X(m))-\tilde{X}(Y(m))=\bar{c}+\widetilde{0}_{Y(p)}(m),
$$

where the subtraction on the left is the usual subtraction of vectors which are tangent to $T M$ at $Y(m)$, and the addition on the right is addition in $T(p): T^{2} M \rightarrow T M$. That is, $\bar{c}+\underset{T(p)}{+\widetilde{0}_{Y(m)}}$ is the vertical lift of $c$ to $Y(m)$ and so, by $(5), c=[X, Y](m)$. Briefly,

$$
T(Y)(X(m))-\tilde{X}(Y(m)) \triangleright[X, Y](m) .
$$

A comment on notation: In the case of a general double vector bundle $D$, the two additions $+{ }_{A}$ and $+_{B}$ are distinct. In the case of $T^{2} M$, however, both side bundles are copies of $T M$. To distinguish between the two structures, we use the projection maps; for example, addition in $T^{2} M \stackrel{T(p)}{\longrightarrow} T M$ will be denoted by $\underset{T(p)}{+}$. We adopt this notation whenever necessary, especially in Sects. 5 and 6.

Note that (5) needs to be proved in local coordinates, or in terms of the action of vector fields on functions. The use of (6) expresses the result in a compact conceptual way.

We now express these results in the terms that will be used throughout the paper. Consider a double vector bundle $D$ as in (1). 
Definition 1 A pair of sections $X \in \Gamma A$ and $\xi \in \Gamma_{B} D$ form a linear section of $D$ if $\xi$ is a morphism of vector bundles over $X$.

A grid on $D$ is a pair of linear sections $(\xi, X)$ and $(\eta, Y)$ as shown in (7).

That nontrivial grids exist on a double vector bundle is ensured by the assumption that $D \rightarrow A \times_{M} B$ has a right-inverse that is linear in both $A$ and $B$; that is, that $D$ admits sigma-maps.

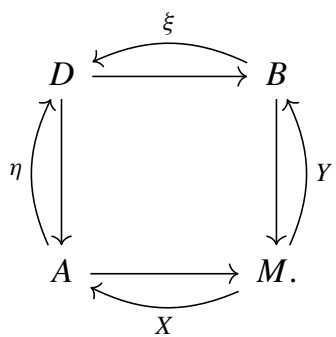

For each $m \in M, \xi(Y(m))$ and $\eta(X(m))$ have the same outline. They therefore determine an element of the core $C$ and, as $m$ varies, a section of $C$ which we denote $\mathrm{w}(\xi, \eta)$. Precisely,

$$
\begin{aligned}
& \xi(Y(m)) \frac{-}{A} \eta(X(m))=\mathrm{w}(\xi, \eta)(m)+{ }_{B} \widetilde{0}_{X(m)}, \\
& \xi(Y(m)) \frac{-}{B} \eta(X(m))=\mathrm{w}(\xi, \eta)(m)+{ }_{A} \widetilde{0}_{Y(m)} .
\end{aligned}
$$

Definition 2 The warp of the grid (7) consisting of $(\xi, X)$ and $(\eta, Y)$ is $\mathrm{w}(\xi, \eta) \in \Gamma C$.

Note that $\mathrm{w}(\xi, \eta)$ changes sign if $\xi$ and $\eta$ are interchanged. Our convention gives the positive sign to the counterclockwise composition $\xi \circ Y$.

The question of signs - or orientations - will haunt us throughout the paper. Later on, we will see that there are various rules that, in many cases, determine which difference to take as the positive warp. These rules generally follow from established conventions of differential geometry.

Equation (5) can now be expressed as saying that the warp of (6) is $[X, Y]$.

Before proceeding, we give two further examples of grids and warps in double vector bundles.

Example 1 Consider the double vector bundle $T A$, the middle diagram in (1), where $(A, q, M)$ is a vector bundle, and let $\nabla$ be a connection in $A$.

Recall the horizontal lifting of vector fields from $M$ to $A$ induced by $\nabla$. To define a vector field on $A$ it is sufficient to define its effect on pullback functions $f \circ q$ for $f \in$ $C^{\infty}(M)$, and on linear functions $\ell_{\varphi}$ for $\varphi \in \Gamma A^{*}$, defined by $\ell_{\varphi}(a)=\langle\varphi(q(a)), a\rangle$.

Given a vector field $Z$ on $M$, denote by $Z^{H}$ the horizontal lift of $Z$ to $A$ defined by

$$
Z^{H}\left(\ell_{\varphi}\right)=\ell_{\nabla_{Z}^{*}(\varphi)}, \quad Z^{H}(f \circ q)=Z(f) \circ q,
$$

where $\nabla^{*}$ is the connection in $A^{*}$ dual to $\nabla$. Since $Z^{H}$ maps linear functions to linear functions and pullbacks to pullbacks, it is a linear vector field; it clearly projects to $Z$. 
The word 'horizontal' here has its standard meaning in connection theory, and does not refer to the structures in $T A$.

It is straightforward to check that $\left(Z_{1}+Z_{2}\right)^{H}=Z_{1}^{H}+Z_{2}^{H}$ and that $(f Z)^{H}=$ $(f \circ q) Z^{H}$.

Now take any $\mu \in \Gamma A$ and form the grid shown in (9).

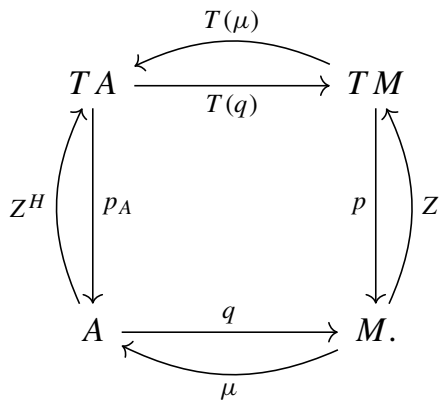

We claim that the warp of the grid is $\nabla_{Z} \mu$; that is, for $m \in M$,

$$
T(\mu)(Z(m))-Z^{H}(\mu(m))=\left(\left(\nabla_{Z} \mu\right)(m)\right)^{\uparrow}(\mu(m)),
$$

where the right-hand side is the vertical lift of $\left(\nabla_{Z} \mu\right)(m) \in A_{m}$ to $T_{\mu(m)} A$.

We give a sketch proof of (10). Again, it is sufficient to verify equality on linear functions and pullbacks. Applying each side of (10) to a pullback $f \circ q, f \in C^{\infty}(M)$, gives zero.

Consider a linear function $\ell_{\varphi}$ for $\varphi \in \Gamma A^{*}$. We have

$$
\begin{aligned}
T(\mu)(Z(m))\left(\ell_{\varphi}\right) & =Z(m)\left(\ell_{\varphi} \circ \mu\right)=Z(m)(\langle\varphi, \mu\rangle) \\
& =\left\langle\nabla_{Z(m)}^{*}(\varphi), \mu(m)\right\rangle+\left\langle\varphi(m), \nabla_{Z(m)}(\mu)\right\rangle,
\end{aligned}
$$

and $Z^{H}(\mu(m))\left(\ell_{\varphi}\right)=\left\langle\nabla_{Z(m)}^{*}(\varphi), \mu(m)\right\rangle$.

Lastly, $\left\langle\varphi(m), \nabla_{Z(m)}(\mu)\right\rangle=\left(\left(\nabla_{Z} \mu\right)(m)\right)^{\uparrow}(\mu(m))\left(\ell_{\varphi}\right)$. For more details see [16, §3.4].

Suppose given a sigma-map $\Sigma: T M \times_{M} A \rightarrow T A$. For $Z \in \mathfrak{X}(M)$ define $Z^{H} \in \mathfrak{X}(A)$ by $Z^{H}(a)=\Sigma(Z(m), a)$ for $a \in A_{m}$. This $Z^{H}$ is a linear vector field over $Z$. Thus a sigma-map defines a horizontal lifting process, and thus a connection in terms of covariant derivatives $\nabla_{Z}$. These processes can be reversed to show that every connection in terms of covariant derivatives $\nabla_{Z}$ defines a sigma-map.

It is thus possible to work with connections in vector bundles by using covariant derivatives, by horizontal lifting processes, or by using sigma-maps for the double vector bundle $T A$. The $\nabla$ formulation is now almost universal. However it does not relate easily to the notion of connection in a principal bundle. Kobayashi and Nomizu [11] gave two global definitions of a connection in a principal bundle $P(M, G)$ : as a suitable $\mathfrak{g}$-valued 1 -form on $P$ and as an invariant horizontal distribution on $P$. The latter defines, and is equivalent to, a lifting of vector fields on $M$ to invariant horizontal 
vector fields on $P$, and this formulation resembles the lifting of vector fields in a vector bundle.

In the 1970s several authors, notably Dieudonné [4] and Besse [2], used the lifting formulation for connections in vector bundles. Dieudonné [4, XVII.16] used what we have called a sigma-map $T M \times_{M} A \rightarrow T A$, and Besse used the corresponding left-split map $T A \rightarrow A$ into the core. Equation (10) may be discerned on page 38 of [2] and is a special case of (17.17.2.1) in [4].

This example is central to Sect. 5. We will consider curvature in Sect. 5.

Example 2 For the third diagram in (1), first consider the manifold $T^{*} A$ where $(A, q, M)$ is a vector bundle. There is a canonical diffeomorphism, denoted $R$, from $T^{*}\left(A^{*}\right)$ to $T^{*} A$, which reverses the standard symplectic structures; see [19] and references given there. We define $r: T^{*} A \rightarrow A^{*}$ to be the composite of $R^{-1}$ with the projection $T^{*}\left(A^{*}\right) \rightarrow A^{*}$. Similarly, use $R^{-1}$ to transport the vector bundle structure of $T^{*}\left(A^{*}\right) \rightarrow A^{*}$ to $T^{*} A \rightarrow A^{*}$. Then the third diagram in (1) is a double vector bundle. The core of $T^{*} A$ can be identified with $T^{*} M$; given $\omega \in T_{m}^{*} M$, its image $\bar{\omega} \in T^{*} A$ is the pullback of $\omega$ across $q: A \rightarrow M$ to $0_{m}^{A}$. The map $R^{-1}$ carries $\bar{\omega} \in T^{*} A$ to $\overline{-\omega} \in T^{*}\left(A^{*}\right)$.

Given a section $\varphi \in \Gamma A^{*}$, the 1 -form $d \ell_{\varphi}: A \rightarrow T^{*} A$ is a linear section over $\varphi$.

Likewise given $\mu \in \Gamma A$, we obtain a 1 -form $d \ell_{\mu}$ on $A^{*}$. Composing with the map $R: T^{*}\left(A^{*}\right) \rightarrow T^{*} A$, we obtain a linear section of $T^{*} A \rightarrow A^{*}$ over $\mu$.

It was proved in [19] that

$$
R\left(d \ell_{\mu}(\varphi(m))\right)-d \ell_{\varphi}(\mu(m))=-q^{*}(d\langle\varphi, \mu\rangle)(\mu(m)) .
$$

(or see $[16,9.5 .3])$. This shows that the warp of the grid which consists of $\left(R \circ\left(d \ell_{\mu}\right), \mu\right)$ and $\left(d \ell_{\varphi}, \varphi\right)$ is $-d\langle\varphi, \mu\rangle$.

\subsection{Outline of the paper}

In a previous paper [18] one of us used a grid in the triple vector bundle $T^{3} M$ to express the Jacobi identity as a statement about the warps of the grids in the constituent double vector bundles. The proof given in that paper relied on a decomposition of $T^{3} M$ into seven copies of $T M$, and it was not clear whether the apparatus of grids and warps had provided a proof of the Jacobi identity or merely a formulation of it. One purpose of the present paper is to give an intrinsic proof of a general result for triple vector bundles and to resolve this question.

Section 2 introduces the basic setup and notation for triple vector bundles. In Sect. 2.2 we formulate the main theorem of the paper on the warps of a grid on a triple vector bundle.

In a double vector bundle two elements with the same outline determine a core element (up to sign). In a triple vector bundle there are intermediate levels at which two elements may have the same outline, and there is more than one notion of core. Section 3 is concerned with describing the core elements determined by pairs of elements for which some levels of the outlines are equal. A brief summary is given at the end of the section. 
Section 4 gives the proof of the warp theorem, Theorem 1. This states, roughly, that given a grid on a triple vector bundle $E$, the sum of the ultrawarps is zero. The ultrawarps are the warps of the grids induced on the core double vector bundles by the grid on $E$. The proof is intrinsic and does not rely on a decomposition of $E$. A brief summary is given at the end of the section.

In Sect. 5 we consider a vector bundle $A$ and the triple vector bundle $T^{2} A$. A connection in $A$ induces a grid in $T^{2} A$, and we show that the concept of curvature arises from the warp theorem; see Theorem 2.

Section 6 presents the example of $T^{3} M$ and the deduction of the Jacobi identity for the Lie bracket of vector fields on $M$ from the warp theorem.

Some concluding remarks are given at the end of the paper.

\section{Triple vector bundles and the warp theorem}

In this section we do two things. First, we set up everything we need for triple vector bundles, in order to formulate the warp theorem (Theorem 1). Secondly, we describe the original formulation [18] of the theorem, and outline the steps which lead to an intrinsic proof.

\subsection{Basics on triple vector bundles}

The basic structure of triple vector bundles has been given in $[9,15,18]$.

Consider the following diagram,

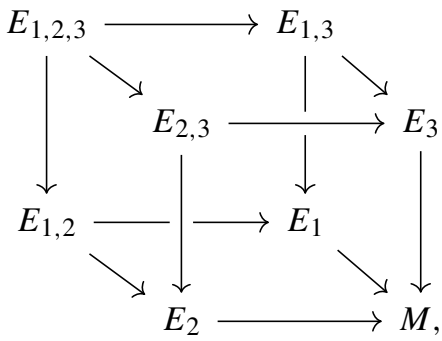

where each edge is a vector bundle. By an upper face we mean a face which has $E_{1,2,3}$ as total space. The lower faces are the three faces which have $M$ as base manifold. We refer to the faces by the names

Back, Front, Left, Right, Up, Down.

The total space of (11) should be denoted, for consistency with the labeling scheme, by $E_{1,2,3}$ but we will usually denote it by $E$.

Definition 3 A cube of vector bundles is a system of vector bundle structures as in (11), such that each face is a double vector bundle, and such that the vector bundle 
operations in $E \rightarrow E_{1,2}$ are morphisms of double vector bundles from the Up face of $E$ to the Down face of $E$ and similarly for the other vector bundle structures in $E$.

The terminology 'cube of vector bundles' is temporary; in $[9,15,18]$ the definition of a triple vector bundle included the (sometimes tacit) requirement that what was there called a decomposition existed, and this can be seen to be equivalent to the existence of a sigma-map. In order to isolate the condition that ensures the existence of a sigmamap, we introduce the above terminology for structures without this condition. In the rest of this subsection we work with such a single cube of vector bundles $E$.

How do we add elements in $E$ ? If $e, f \in E$ lie over the same point of $E_{2,3}$, as shown in (12), their sum has the outline shown in (13).
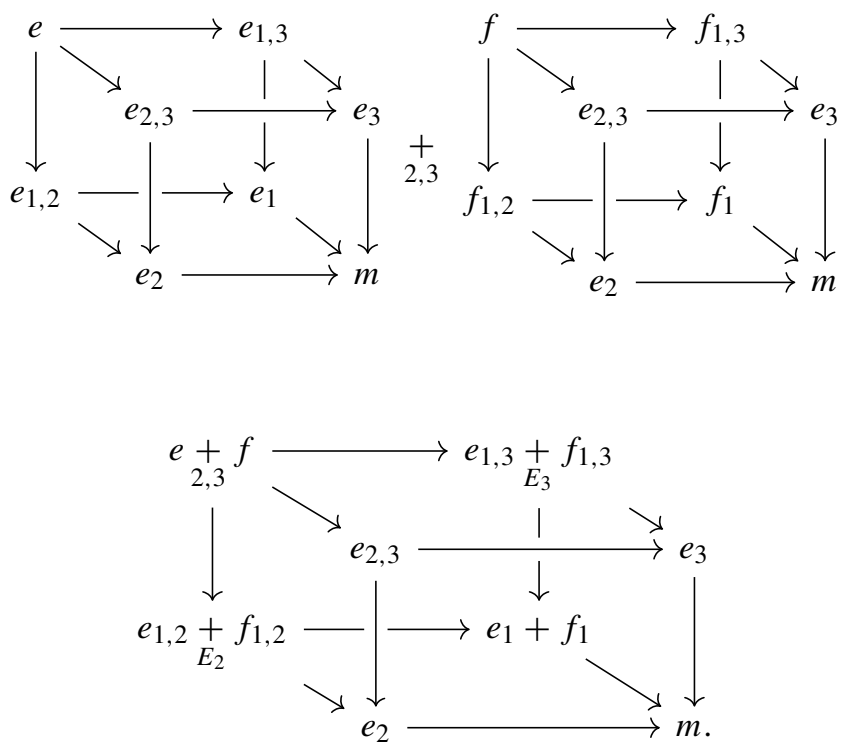

The outlines for scalar multiplication are similar.

Core double vector bundles and the ultracore. Since each face of $E$ is a double vector bundle, each face has a core vector bundle.

The cores of the lower faces $E_{i, j}$ are denoted $E_{i j}$ with the comma removed. The core of the upper face with base manifold $E_{k}$ is denoted $E_{i j, k}$. (This convention comes from [8].)

Focus on the core vector bundles of the Up and of the Down faces. The Up face projects to the Down face via the double vector bundle morphism which consists of the bundle projections $E_{1,2,3} \rightarrow E_{1,2}, E_{2,3} \rightarrow E_{2}, E_{1,3} \rightarrow E_{1}$ and $E_{3} \rightarrow M$. The restriction of $E_{1,2,3} \rightarrow E_{1,2}$ to $E_{12,3}$ goes into $E_{12}$ and inherits the vector bundle structure of $E_{1,2,3} \rightarrow E_{1,2}$. Together with the vector bundle structures on the cores of the Up face and the Down face, this yields another double vector bundle, with total space $E_{12,3}$, which we call the $(U-D)$ core double vector bundle. 
Of course this can also be done for the other two pairs of parallel faces. So there are three core double vector bundles, shown in (14).
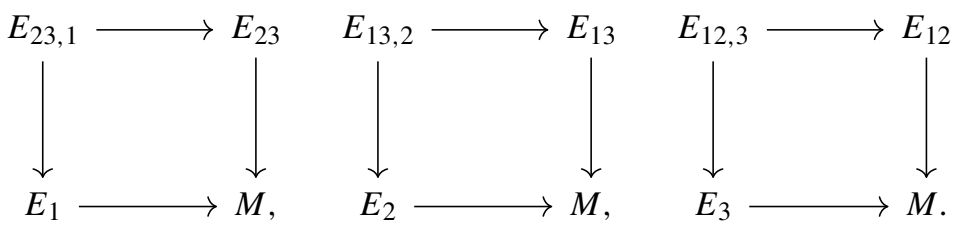

Elements of the core of $E_{12,3}$ project to zeros in the Down face. In the Up face they project to zeros over the zero in $E_{3}$. It follows that an element of the core of $E_{12,3}$ projects to zero in every bundle structure. Equally the cores of the (B-F) and (L-R) double vector bundles consist of the elements of $E_{1,2,3}$ which project to zeros in every bundle structure. Thus each double vector bundle in (14) has the same core. This is denoted $E_{123}$ (without commas) and called the ultracore of $E$.

From the interchange laws it follows that the three additions on $E$, namely,,,+++ and + , coincide on the ultracore and give it the structure of a vector bundle over $M$. 2,3

Notation for zero sections. The zero section of $E_{1}$ is denoted by $0^{E_{1}}: M \rightarrow E_{1}$, $m \mapsto 0_{m}^{E_{1}}$, with similar notations for $E_{2}$ and $E_{3}$.

The zero section of $E_{1,2} \rightarrow E_{1}$ is denoted by $\tilde{0}^{1,2}: E_{1} \rightarrow E_{1,2}, e_{1} \mapsto \tilde{0}_{e_{1}}^{1,2}$. The double zero of $E_{1,2}$ is denoted by $\odot_{m}^{1,2}$, with similar notations for the other vector bundle structures.

The zero section of $E \rightarrow E_{1,2}$ is denoted by $\hat{0}: E_{1,2} \rightarrow E, e_{1,2} \mapsto \hat{0}_{e_{1,2}}$. Note that the subscripts of the element $e_{1,2}$ are enough to indicate that this is the zero section of $E$ over $E_{1,2}$; there is no need for superscripts on $\hat{0}$.

Finally, the triple zero of $E$ is denoted by $\odot_{m}^{3}$. This is the zero of the ultracore vector bundle.

Notation for projections. The superscripts of the projection maps of a cube of vector bundles $E$ denote the domain, and the subscripts denote the target, for example, $q_{2}^{1,2}$ : $E_{1,2} \rightarrow E_{2}$. We omit the corresponding script when the domain is $E$, for example $q_{1,2}: E \rightarrow E_{1,2}$, or the target is $M$, for example $q^{2}: E_{2} \rightarrow M$.

Instances of the interchange law. As mentioned in the Introduction Eq. (3) is one of the interchange laws for double vector bundles. In the triple vector bundle setting, since $E$ has three different vector bundle structures, we have interchange laws relating either two, or all three vector bundle structures. In this paper we only need the interchange laws that relate two of the three vector bundle structures.

Without loss of generality, focus on the Left face. This is a double vector bundle. The interchange law in the Left face for four elements $e, f, g, h \in E$, over the same 
$e_{2} \in E_{2}$ is the following,

$$
\underset{2,3}{(e+f} \underset{1,2}{+}(\underset{2,3}{+} h)=(\underset{1,2}{+} g) \underset{2,3}{+}(\underset{1,2}{+} h)
$$

where the outlines of the four elements in question are:
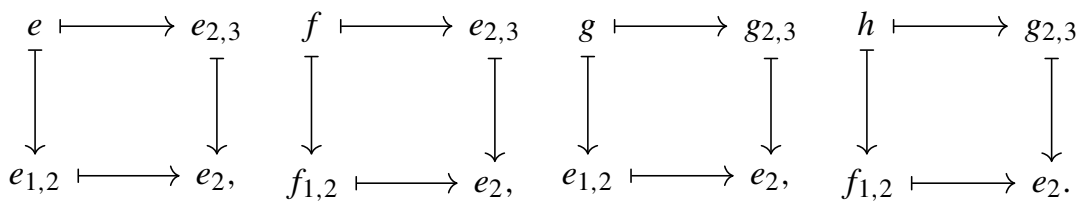

Recall that the projection map from the Left to the Right face is a double vector bundle morphism. Consequently, the interchange law in the Right face holds for the projections of the four elements $e, f, g, h \in E$ in $E_{1,3}$ : namely $e_{1,3}, f_{1,3}, g_{1,3}$, and $h_{1,3}$ :

$$
\left(e_{1,3}+\underset{E_{3}}{+} f_{1,3}\right)+\underset{E_{1}}{+}\left(g_{1,3}+\underset{E_{3}}{+} h_{1,3}\right)=\left(e_{1,3}+g_{E_{1}} g_{1,3}\right) \underset{E_{3}}{+}\left(f_{1,3}+h_{E_{1}} h_{1,3}\right),
$$

with outlines
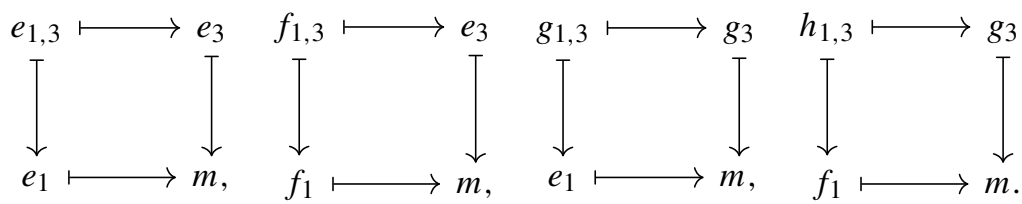

\section{Result of Li-Bland and Ševera for cubes of vector bundles}

In [13] Li-Bland and Ševera proved that the condition in the definition of a double vector bundle $D$ on page 2, that the double source map $\sharp: D \rightarrow A \times{ }_{M} B$ is a surjective submersion, actually follows from the other conditions.

We now prove the corresponding result for cubes of vector bundles. We first need to show that the set that corresponds to $A \times{ }_{M} B$ is a manifold.

Lemma 1 Given a cube of vector bundles $E$, write $W$ for the set of all $\left(e_{1,2}, e_{2,3}, e_{1,3}\right)$ in $E_{1,2} \times E_{2,3} \times E_{1,3}$ such that

$$
q_{2}^{1,2}\left(e_{1,2}\right)=q_{2}^{2,3}\left(e_{2,3}\right), \quad q_{3}^{2,3}\left(e_{2,3}\right)=q_{3}^{1,3}\left(e_{1,3}\right), \quad q_{1}^{1,3}\left(e_{1,3}\right)=q_{1}^{1,2}\left(e_{1,2}\right)
$$

Then $W$ is a closed embedded submanifold of $E_{1,2} \times E_{2,3} \times E_{1,3}$.

Note: This result is valid for three double vector bundles arranged as are the three lower faces of $E$. In the terminology of [15], the result holds for cornerings. 
Proof First define $F: E_{1,2} \times E_{2,3} \times E_{1,3} \rightarrow E_{2} \times E_{2} \times E_{3} \times E_{3} \times E_{1} \times E_{1}$ by

$$
\begin{aligned}
& F\left(e_{1,2}, e_{2,3}, e_{1,3}\right) \\
& \quad=\left(q_{2}^{1,2}\left(e_{1,2}\right), q_{2}^{2,3}\left(e_{2,3}\right), q_{3}^{2,3}\left(e_{2,3}\right), q_{3}^{1,3}\left(e_{1,3}\right), q_{1}^{1,3}\left(e_{1,3}\right), q_{1}^{1,2}\left(e_{1,2}\right)\right) .
\end{aligned}
$$

This is (a rearrangement of) the product of three surjective submersions. Define

$$
\Delta=\left\{\left(e_{2}, e_{2}, e_{3}, e_{3}, e_{1}, e_{1}\right) \mid e_{2} \in E_{2}, e_{3} \in E_{3}, e_{1} \in E_{1}\right\}
$$

This $\Delta$ is a submanifold of the target of $F$ and $F$ is a surjective submersion, so $F^{-1}(\Delta)$ is a submanifold of $E_{1,2} \times E_{2,3} \times E_{1,3}$.

We claim that $F^{-1}(\Delta)=W$. Note that if $\left(e_{1,2}, e_{2,3}, e_{1,3}\right) \in F^{-1}(\Delta)$ then all three elements project to the same element of $M$. Given $\left(e_{1,2}, e_{2,3}, e_{1,3}\right) \in F^{-1}(\Delta)$, write $e_{1}, e_{2}, e_{3}$ as above and write $m=q^{1}\left(e_{1}\right)$. Then

$$
q^{3}\left(e_{3}\right)=q^{3}\left(q_{3}^{1,3}\left(e_{1,3}\right)\right)=q^{1}\left(q_{1}^{1,3}\left(e_{1,3}\right)\right)=q^{1}\left(e_{1}\right)=m
$$

Likewise $q^{2}\left(e_{2}\right)=m$. So $\left(e_{1,2}, e_{2,3}, e_{1,3}\right) \in W$. This completes the proof of the Lemma.

The triple source map of the cube of vector bundles $E$, denoted $\widetilde{G}$, is the map $E \rightarrow W$ formed from the bundle projections of $E$; that is,

$$
\widetilde{\natural}(e)=\left(q_{1,2}(e), q_{2,3}(e), q_{1,3}(e)\right) .
$$

Now let us denote by

$$
\natural^{\mathrm{B}}: E \rightarrow E_{1,2} \times_{E_{1}} E_{1,3}, \quad \natural^{\mathrm{F}}: E_{2,3} \rightarrow E_{2} \times_{M} E_{3}
$$

the double source maps for the Back face and for the Front face of $E$, respectively.

Proposition 1 If $E$ is a cube of vector bundles as in (11), then the triple source map $\tilde{\natural}: E \rightarrow W$ is a surjective submersion.

Proof The key to the proof is the following commutative diagram. Note that $\iota^{\mathrm{B}}$ is a morphism of vector bundles over $\iota^{\mathrm{F}}$; (17) is not a double structure.

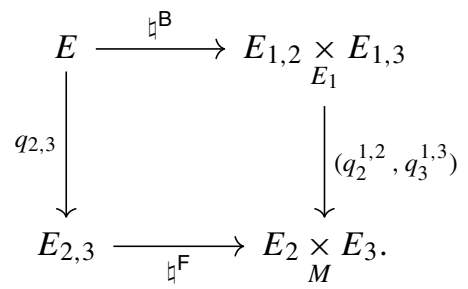


Take any $e_{2,3} \in E_{2,3}$, with $\iota^{\mathrm{F}}\left(e_{2,3}\right)=\left(e_{2}, e_{3}\right)$. Using the notation for the zero section of $E \rightarrow E_{2,3}$ as introduced earlier, write $\left.\hat{0}_{e_{2,3}} \in E\right|_{e_{2,3}}$. The decompositions of the following tangent spaces are a standard result of vector bundle theory:

$$
\begin{aligned}
T_{\hat{0}_{e_{2,3}}} E & =\left.E\right|_{e_{2,3}} \oplus T_{e_{2,3}}\left(E_{2,3}\right), \\
T_{\left(\tilde{0}_{e_{2}}^{1,2}, \tilde{0}_{e_{3}}^{1,3}\right)}\left(E_{1,2} \underset{E_{1}}{\times} E_{1,3}\right) & =\left(E_{1,2}^{\left.\underset{E_{1}}{\times} E_{1,3}\right)\left.\right|_{\left(e_{2}, e_{3}\right)}} \oplus T_{\left(e_{2}, e_{3}\right)}\left(E_{2} \underset{M}{\times} E_{3}\right) .\right.
\end{aligned}
$$

Take the tangent of the vector bundle morphism (17) at point $\hat{0}_{e_{2,3}}$. This decomposes into

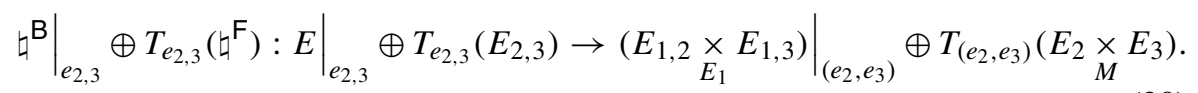

To see the first component of this decomposition, note that $T_{\hat{0}_{e_{2,3}}}\left(\mathfrak{\natural}^{\mathrm{B}}\right)$ maps the vertical tangent vectors of $E$ at $\hat{0}_{e_{2,3}}$, which can be canonically identified with $\left.E\right|_{e_{2,3}}$, to the vertical tangent vectors of $E_{1,2} \underset{E_{1}}{\times} E_{1,3}$ at $\left(\tilde{0}_{e_{2}}^{1,2}, \tilde{0}_{e_{3}}^{1,3}\right)$, which can likewise be identified with $\left.\left(E_{1,2} \underset{E_{1}}{\times} E_{1,3}\right)\right|_{\left(e_{2}, e_{3}\right)}$.

In other words, for a $\xi \in T_{\hat{0}_{e_{2,3}}} E$ with $T_{\hat{0}_{e_{2,3}}}\left(q_{2,3}\right)(\xi)=0_{e_{2,3}}^{T\left(E_{2,3}\right)}$, it follows that

$$
T_{\left(\tilde{0}_{e_{2}}^{1,2}, \tilde{0}_{e_{3}}^{1,3}\right)}\left(q_{2}^{1,2}, q_{3}^{1,3}\right)\left(T_{\hat{0}_{e_{2,3}}}\left(\bigsqcup^{\mathrm{B}}\right)(\xi)\right)=\left(0_{e_{2}}^{T\left(E_{2}\right)}, 0_{e_{3}}^{T\left(E_{3}\right)}\right),
$$

from the commutativity of the diagram (17). Here $\left(0_{e_{2}}^{T\left(E_{2}\right)}, 0_{e_{3}}^{T\left(E_{3}\right)}\right)$ denotes the zero in $T_{\left(e_{2}, e_{3}\right)}\left(E_{2} \underset{M}{\times} E_{3}\right)$.

Map (20) is surjective since by hypothesis $\sharp^{B}$ is a surjective submersion (as the Back face is a double vector bundle). Therefore, the first component of (20) is surjective. Consequently, for any $\left(e_{1,2}, e_{2,3}, e_{1,3}\right) \in W$, there exists an $\left.e \in E\right|_{e_{2,3}}$ such that $q_{1,2}(e)=e_{1,2}$, and $q_{1,3}(e)=e_{1,3}$.

By applying the tangent functor to (11) (we do not need to consider the hypercube structure), and applying the same argument to the resulting structure, we see that the triple source map is a submersion.

This completes the proof of Proposition 1.

The result of Li-Bland and Ševera on which Proposition 1 is based was established for $\mathscr{V} \mathscr{B}$-Lie groupoids rather than double vector bundles. By combining the techniques of their proof and that above, we could establish a similar result for $\mathscr{V} \mathscr{B}$-double Lie groupoids.

We can now state the definition of a triple vector bundle. 
Definition 4 A triple vector bundle is a cube of vector bundles for which there exists a morphism of cubes of vector bundles $\widetilde{\Sigma}: W \rightarrow E$ that is right-inverse to the triple source map $E \rightarrow W$.

It seems likely that the existence of sigma-maps follows from the definition of a cube of vector bundles (compare [6]) or by using Proposition 1, but we prefer to state it as an explicit assumption.

Grids on triple vector bundles. A grid in a double vector bundle constitutes two linear sections. In a triple vector bundle the concept of grid requires what we call linear double sections.

Definition 5 A down-up linear double section of $E$ is a collection of sections

$$
Z_{1,2}: E_{1,2} \rightarrow E_{1,2,3}, \quad Z_{1}: E_{1} \rightarrow E_{1,3}, \quad Z_{2}: E_{2} \rightarrow E_{2,3}, \quad Z: M \rightarrow E_{3},
$$

which form a morphism of double vector bundles from the Down face to the Up face.

The core morphism of $Z_{1,2}$ defines a vector bundle morphism from the core of the Down face to the core of the Up face. We denote this by $Z_{12}: E_{12} \rightarrow E_{12,3}$. It is a linear section over $Z: M \rightarrow E_{3}$.

In a similar fashion we define right-left and front-back linear double sections of $E$.

Definition 6 A grid on $E$ is a set of three linear double sections, one in each direction, as shown in (21).

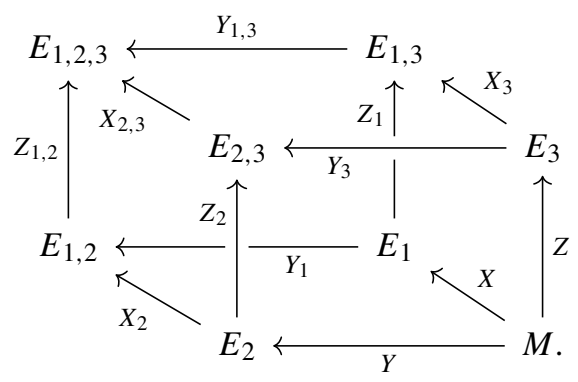

That nontrivial grids exist is guaranteed by the existence of sigma-maps; more precisely, a grid exists for any given sections $X, Y, Z$.

We note the following equations for future reference. They follow from the fact that the double sections are morphisms of double vector bundles.

For $e_{1,2}, e_{1,2}^{\prime}$ over the same point of $E_{1}$,

$$
Z_{1,2}\left(e_{1,2} \frac{-e_{1}}{E_{1,2}}\right)=Z_{1,2}\left(e_{1,2}\right) \frac{-}{1,3} Z_{1,2}\left(e_{1,2}^{\prime}\right) .
$$

For $e_{1,2}, e_{1,2}^{\prime}$ over the same point of $E_{2}$,

$$
Z_{1,2}\left(e_{1,2} \frac{-}{E_{2}} e_{1,2}^{\prime}\right)=Z_{1,2}\left(e_{1,2}\right) \frac{-}{2,3} Z_{1,2}\left(e_{1,2}^{\prime}\right)
$$




\subsection{Formulation of the warp theorem}

We now have everything we need in order to describe the original formulation of the theorem, as given in [18].

Start with a grid on $E$. Focus on the Up face of the triple vector bundle. We see that $\left(Y_{1,3}, Y_{3}\right)$ and $\left(X_{2,3}, X_{3}\right)$ define a grid on the Up face. Denote its warp by $\mathrm{w}_{\mathrm{up}}$. This is a section of the core vector bundle of the Up face, that is, $\mathrm{w}_{\mathrm{up}}: E_{3} \rightarrow E_{12,3}$. Similarly for the Down face, its warp $\mathrm{w}_{\text {down }}$ is a section of the core vector bundle of the Down face, so $\mathrm{w}_{\text {down }}: M \rightarrow E_{12}$. It follows that ( $\mathrm{w}_{\mathrm{up}}, \mathrm{w}_{\mathrm{down}}$ ) is a linear section of the (U-D) core double vector bundle. Recall that the core morphism $Z_{12}$ of the linear double section $Z_{1,2}$ defines another linear section of the (U-D) core double vector bundle. Therefore, we have the following grid on $E_{12,3}$ :

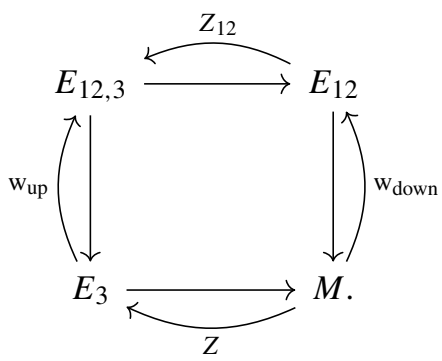

We call the warp of this grid the Up-Down ultrawarp and denote it by uUD. It is a section of the ultracore $E_{123}$.

Of course we can also build corresponding grids on the other two core double vector bundles. We therefore have three ultrawarps, as shown in (24).

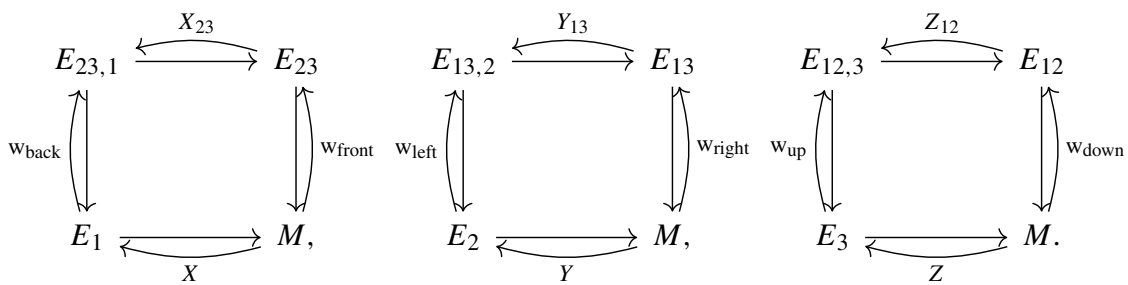

We take the ultrawarps with the orientations opposite to (24); that is, using the $\triangleright$ notation,

$$
\begin{aligned}
& \mathrm{w}_{\text {back }} \circ X-X_{23} \circ \mathrm{w}_{\text {front }} \triangleright \mathrm{u}_{\mathrm{BF}}, \\
& \mathrm{w}_{\text {left }} \circ Y-Y_{13} \circ \mathrm{w}_{\text {right }} \triangleright \mathrm{u}_{\mathrm{LR}}, \\
& \mathrm{w}_{\text {up }} \circ Z-Z_{12} \circ \mathrm{w}_{\text {down }} \triangleright \mathrm{u}_{\mathrm{UD}} .
\end{aligned}
$$

We can now state the main theorem about grids on triple vector bundles. 
Theorem 1 (Warp Theorem) Given a triple vector bundle $E$ and a grid on $E$ as in (21),

$$
\mathrm{u}_{\mathrm{BF}}+\mathrm{u}_{\mathrm{LR}}+\mathrm{u}_{\mathrm{UD}}=0 .
$$

To give an intrinsic proof, we need to describe the ultrawarps in an alternative way.

So far, the only equation we have seen that describes the warp of a grid on a double vector bundle is (8). Focus on the ultrawarp uUD. From the grid on the (U-D) core double vector bundle, for $m \in M$, by (8) we have that

$$
\left(\mathrm{w}_{\mathrm{up}} \circ Z\right)(m) \frac{-}{2,3}\left(Z_{12} \circ \mathrm{w}_{\mathrm{down}}\right)(m)=\hat{0}_{Z(m)}+\mathrm{u}_{1,2} \mathrm{UD}(m) .
$$

How can we express $\left(\mathrm{w}_{\mathrm{up}} \circ Z\right)(m)$ and $\left(Z_{12} \circ \mathrm{w}_{\text {down }}\right)(m)$ in a more useful way? About $\mathrm{w}_{\mathrm{up}}$, for any $e_{3} \in E_{3}$, again from (8) we have that

$$
Y_{1,3}\left(X_{3}\left(e_{3}\right)\right) \frac{-}{1,3} X_{2,3}\left(Y_{3}\left(e_{3}\right)\right)=\hat{0}_{X_{3}\left(e_{3}\right)}+\mathrm{w}_{\mathrm{up}}\left(e_{3}\right) .
$$

Putting $e_{3}=Z(m)$, we have

$$
Y_{1,3}\left(X_{3}(Z(m))\right) \frac{-}{1,3} X_{2,3}\left(Y_{3}(Z(m))\right)=\hat{0}_{X_{3}(Z(m))}+{ }_{2,3}^{+} \mathrm{w}_{\mathrm{up}}(Z(m)) .
$$

We introduce a more succinct notation, for use in calculations.

$$
\begin{aligned}
& \mathrm{ZYX}=Z_{1,2}\left(Y_{1}(X(m))\right), \mathrm{YZX}=Y_{1,3}\left(Z_{1}(X(m))\right), \mathrm{XZY}=X_{2,3}\left(Z_{2}(Y(m))\right), \\
& \mathrm{ZXY}=Z_{1,2}\left(X_{2}(Y(m))\right), \mathrm{YXZ}=Y_{1,3}\left(X_{3}(Z(m))\right), \mathbf{X Y Z}=X_{2,3}\left(Y_{3}(Z(m))\right) .
\end{aligned}
$$

Now (28) becomes

$$
\mathrm{YXZ} \frac{\mathrm{T}, 3}{\mathrm{XYZ}}=\hat{0}_{e_{1,3}^{\prime}}+\lambda_{2,3}
$$

where $e_{1,3}^{\prime}=X_{3}(Z(m))$ and $\lambda_{3}=\mathrm{w}_{\mathrm{up}}(Z(m))$. Note the following. In the case of a double vector bundle, we can rewrite $(8)$ as

$$
\mathrm{w}(\xi, \eta)(m)=\left((\xi \circ Y)(m) \frac{-}{A}(\eta \circ X)(m)\right) \frac{-}{B} 0_{X(m)}^{D} .
$$

In the case of a triple vector bundle, this is also possible. If we tried a similar calculation on (30), since $\hat{0}_{e_{1,3}^{\prime}}=\hat{0}_{e_{1,3}^{\prime}}=\hat{0}_{e_{3}}$, we would have

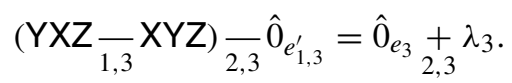

Since $\hat{0}_{e_{3}}$ is the double zero of the Up face over $e_{3}$, we have $\hat{0}_{e_{3}}+\lambda_{2,3}=\lambda_{3}$. So in total, we can rewrite (30) as

$$
\lambda_{3}=\left(\mathrm{YXZ} \frac{\mathrm{XY}}{1,3} \mathrm{XZ}\right) \underset{2,3}{-\hat{0}_{e_{1,3}^{\prime}}} .
$$


About $\left(Z_{1,2} \circ \mathrm{w}_{\text {down }}\right)(m)$, first write $\mathrm{w}_{\text {down }}(m)$ out using $(8)$ as

$$
Y_{1}(X(m)) \underset{E_{1}}{-} X_{2}(Y(m))=\tilde{0}_{X(m)}^{1,2} \underset{E_{2}}{+} \mathrm{w}_{\text {down }}(m) .
$$

Apply $Z_{1,2}$ to this, and using (22) and (23) it follows that

$$
Z_{1,2}\left(Y_{1}(X(m))\right) \frac{-}{1,3} Z_{1,2}\left(X_{2}(Y(m))\right)=\hat{0}_{Z_{1}(X(m))}+Z_{2,3}\left(\mathrm{w}_{\text {down }}(m)\right)
$$

Again, for reasons of economy of space, rewrite this as

$$
Z Y X \frac{Z X Y}{1,3}=\hat{0}_{e_{1,3}}+k_{2,3}
$$

where $e_{1,3}=Z_{1}(X(m))$ and $k_{3}=Z_{12}\left(\mathrm{w}_{\text {down }}(m)\right)$. Alternatively, as in the case of $\lambda_{3}$,

$$
k_{3}=\left(\mathrm{ZYX} \frac{\mathrm{ZXY}}{1,3} \underset{2,3}{-\hat{0}_{e_{1,3}}} .\right.
$$

Let us go back to (27). We can rewrite this as

$$
\lambda_{3} \frac{-3,3}{2,3}=\hat{0}_{e_{3}}+\mathrm{u}_{1,2} \mathrm{UD}(m),
$$

and using (31) and (32), we have that

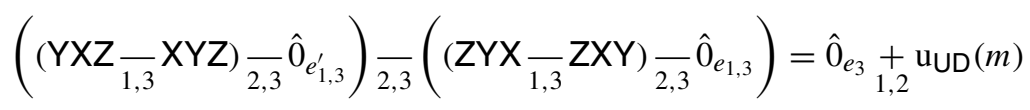

or, more elegantly, using interchange laws,

$$
\begin{aligned}
& \left(Y X Z \frac{1,3}{-X Y Z}\right) \underset{2,3}{-}(\mathrm{YYX} \underset{1,3}{-} \mathrm{ZXY})=\left(\hat{0}_{e_{1,3}^{\prime}}+\lambda_{2,3}\right) \frac{-}{2,3}\left(\hat{0}_{e_{1,3}}+k_{2,3}\right) \\
& =\left(\hat{0}_{e_{1,3}^{\prime}}-\hat{0}_{2,3} e_{1,3}\right)+\left(\lambda_{3} \frac{-3}{2,3} k_{3}\right)=\left(\hat{0}_{e_{1,3}^{\prime}}-\hat{0}_{2,3} e_{1,3}\right)+\left(\hat{0}_{e_{3}}+{ }_{1,2}+\mathrm{uDD}(m)\right) \text {. }
\end{aligned}
$$

In calculations it is generally preferable to use equations of the form (30), and to avoid equations of the form (31).

Therefore, in order to describe ultrawarps such as $\mathrm{UUD}_{\mathrm{D}}(m)$, we will use equations of the form (33), and in abbreviated notation,

$$
(\mathrm{YXZ}-\mathrm{XYZ})-(\mathrm{ZYX}-\mathrm{ZXY}) \triangleright \mathrm{u} \mathrm{UD}(m)
$$

as introduced after (4).

It is worth emphasizing that the above arguments rely on the fact that core and ultracore elements are uniquely determined by equations such as (8). 
There are similar abbreviated equations for the other two ultrawarps. Altogether we have

$$
\begin{aligned}
& (Z Y X-Y Z X)-(X Z Y-X Y Z) \triangleright u_{\mathrm{BF}}(m), \\
& (X Z Y-Z X Y)-(Y X Z-Y Z X) \triangleright u_{\mathrm{LR}}(m), \\
& (Y X Z-X Y Z)-(Z Y X-Z X Y) \triangleright u_{U D}(m),
\end{aligned}
$$

and from now on we will use a further shortening of the notation,

$$
\mathrm{u}_{\mathrm{BF}}(m)=u_{1}, \quad \mathrm{u}_{\mathrm{LR}}(m)=u_{2}, \quad \mathrm{u}_{\mathrm{UD}}(m)=u_{3} .
$$

The main difficulty in proving (26) is that we cannot simply add and subtract the expressions in (34), since the operations are in different vector bundle structures. The apparatus of the next section overcomes this difficulty.

Orientation. A further problem arises from the fact that the warp of a grid on a double vector bundle is only defined up to sign. We now need to consider how to choose these signs consistently for a grid on a triple vector bundle. This is a question of fixing orientations.

We choose to orient each upper face so that the positive term in the formula for the warp defines the outward normal by the right-hand rule. We then take the positive and negative terms in the opposite lower face to match those in the upper face; that is, we orient the lower faces so that the positive term in the warp defines the inward normal.

Thus the orientation of the Up face determines the signs in the first subtraction in (34c) below and the orientation of the Down face determines the signs in the second subtraction.

The "middle subtractions" in (34), that is, the orientations of the core double vector bundles, is an independent choice, equivalent to the choice of signs in (25). What matters here is consistency: if we took all three ultrawarps with the opposite signs, that would also be fine.

\section{Preliminaries for the proof of the warp theorem}

This section contains the main technical work needed for the proof of the warp theorem. We first describe our approach.

We want to find how the three ultrawarps are related, more specifically, for $m \in M$, we want to find a relation between three ultracore elements, $u_{1}, u_{2}, u_{3}$. The best way to do this, where slightly easier calculations are involved, is to manipulate the differences of the six elements, (34a), (34b), and (34c). That is exactly what we do in this and the following section. In this section we obtain formulas expressing the difference of two elements of a triple vector bundle in terms of core elements and zeros. If two elements of a triple vector bundle can be subtracted, then their outlines must have at least one face in common. Cases where the outlines have two or more faces in common arise repeatedly in the rest of the paper. Each of these cases needs individual treatment. 
Looked at from another point of view, two elements of a triple vector bundle which can be subtracted may admit exactly one, or two, or all three, of the subtractions $\frac{-1,2}{\text {, }}$ $\frac{-}{1,3}$, and $\overline{2,3}$.

\subsection{First case: two elements that have the same outline}

Let $e$ and $e^{\prime}$ have exactly the same outline
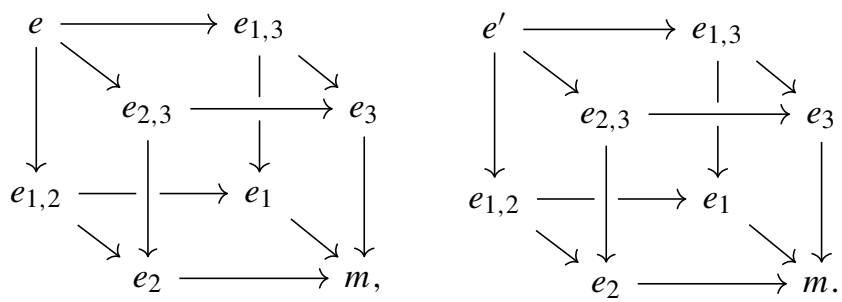

All three differences $e-\frac{e_{1,2}^{\prime}}{e} e \frac{-}{1,3} e^{\prime}, e \frac{-}{2,3} e^{\prime}$ are defined.

Step 1. Focus on the Back faces of $e$ and $e^{\prime}$

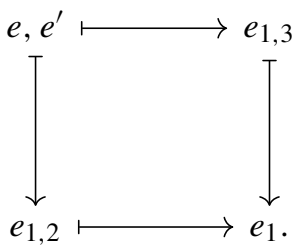

Then, from double vector bundle theory, we can write

$$
e \overline{1,2} e^{\prime}=k_{1}+\hat{0}_{e_{1,2}}, \quad e-\frac{1,3}{\prime} e^{\prime}=k_{1}+\hat{0}_{e_{1,3}},
$$

where $k_{1} \in E_{23,1}$, the core of the Back face, with outline

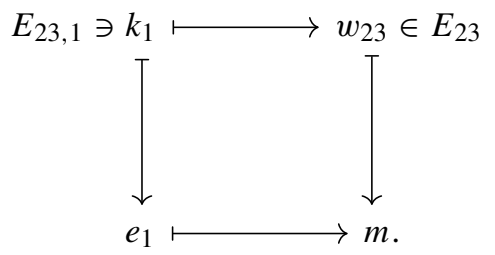

Step 2. Show that $w_{23}=\odot_{m}^{2,3}$. 
Use the morphism $q_{2,3}: E \rightarrow E_{2,3}$. We know that $q_{2,3}\left(e-\frac{e_{1,3}}{}\right)=\tilde{0}_{e_{3}}^{2,3}$ and

$$
q_{2,3}\left(k_{1}+\hat{0}_{1,2} e_{1,3}\right)=q_{2,3}\left(k_{1}\right)+q_{E_{2}} q_{2,3}\left(\hat{0}_{e_{1,3}}\right)=w_{23}+\tilde{E}_{2} \tilde{0}_{e_{3}}^{2,3} .
$$

Therefore

$$
w_{23}+\underset{E_{2}}{+\tilde{0}_{e_{3}}^{2,3}}=\tilde{0}_{e_{3}}^{2,3}
$$

and, from double vector bundle theory, we have that $w_{23}=\underset{0_{m}}{\tilde{E}_{2}}=\odot_{m}^{2,3}$. So $k_{1}$ has the outline

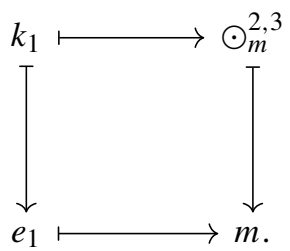

Step 3. Applying double vector bundle theory again, we get

$$
k_{1}=u_{1}+\hat{0}_{2,3},
$$

where $u_{1}$ is an ultracore element.

Step 4. Apply the same procedure to Left and Up faces of $e$ and $e^{\prime}$.

Focus on the Left faces of $e$ and $e^{\prime}$

$$
e \overline{2,3} e^{\prime}=k_{2}+\hat{0}_{e_{2,3}}, \quad e \overline{1,2} e^{\prime}=k_{2,3}+\hat{0}_{e_{1,2}},
$$

where $k_{2} \in E_{13,2}$, core of the Left face, with outline

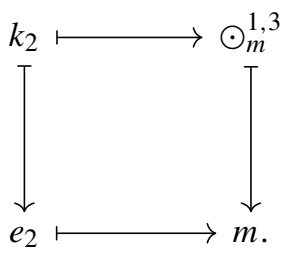

So, we can write

$$
k_{2}=u_{2}+\hat{0}_{e_{2}},
$$

where $u_{2}$ is an ultracore element. Similarly for the Up faces, we have

$$
e-e^{\prime}=k_{3,3}+\hat{0}_{2,3} e_{1,3}, \quad e-e^{\prime}=k_{3}+\hat{0}_{e_{2,3}, 3},
$$


where $k_{3} \in E_{12,3}$, core of the Up face, with outline

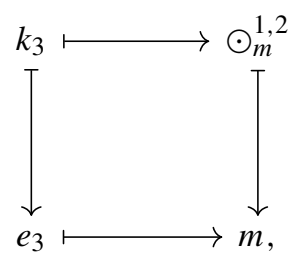

so $k_{3}=u_{3}+\hat{0}_{e_{3}}$ with $u_{3}$ an ultracore element.

Step 5. Show that $u_{1}=u_{2}=u_{3}$.

We show that $u_{1}=u_{3}$. So far, we have two expressions for $e-\frac{1}{1,3} e^{\prime}$, namely:

$$
k_{1}+\hat{0}_{e_{1,3}}=k_{3}+\hat{0}_{e_{1,3}} .
$$

Expand the left-hand side of (35), mimicking the double vector bundle case:

$$
\begin{aligned}
\hat{0}_{e_{1,3}}+\left(\hat{0}_{e_{1}}+u_{1,2}\right) & =\left(\hat{0}_{e_{1,3}}+\hat{0}_{e_{3}}\right)+\left(\hat{0}_{e_{1}}+u_{1}\right) \\
& =\left(\hat{0}_{e_{1,3}}+\hat{0}_{e_{1}}\right)+\left(\hat{0}_{e_{3}}+u_{1,3}\right)=\hat{0}_{e_{1,3}}+\left(\hat{0}_{e_{3}}+u_{1,2}\right) .
\end{aligned}
$$

Therefore, we see that (35) can be rewritten as:

$$
\hat{0}_{e_{1,3}}+\left(\hat{0}_{e_{3}}+u_{1,2}\right)=\hat{0}_{e_{1,3}}+\left(\hat{0}_{e_{3}, 3}+u_{3,2}\right)
$$

from where it follows that $u_{1}=u_{3}$. Similarly, we can show that $u_{2}=u_{3}$.

At this point write $u_{1}=u_{2}=u_{3}$ to be $u$.

Step 6. We obtain six formulas for the differences between $e$ and $e^{\prime}$.

Proposition 2 With the above notation, two elements $e$ and $e^{\prime}$ which have the same outline are related by

$$
\begin{aligned}
& e-e^{\prime}=\hat{0}_{e_{1,3}}+\left(\hat{0}_{e_{1}}+u\right)=\hat{0}_{2,3}+\left(\hat{0}_{e_{1,3}}+u\right), \\
& \left.e-e_{2,3}+u\right)=\hat{0}_{e_{1,2}}+\left(\hat{0}_{e_{1}}+u\right)=\hat{0}_{e_{1,2}}+\left(\hat{0}_{e_{2}}+u\right), \\
& e-e_{1,3}+u e_{1,3}^{\prime}=\hat{0}_{e_{2,3}}+\left(\hat{0}_{e_{3}}+u\right)=\hat{0}_{e_{2,3}}+\left(\hat{0}_{e_{2}}+u\right) .
\end{aligned}
$$

What is important here is that the subtraction with respect to each structure results in the same ultracore element $u$.

We will use the following special case later on. 
Example 3 If $e, e^{\prime}$ are in one of the core double vector bundles, for example if $e, e^{\prime} \in$ $E_{23,1}$, with outline

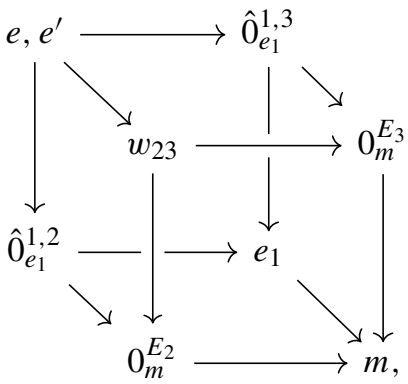

then from (36) we have

$$
e-e^{\prime}=\hat{0}_{w_{23}}+\left(\hat{0}_{1,3}^{E_{3}}+u\right)=\hat{0}_{w_{23}}+\underset{1,3}{+}\left(\odot_{m}^{3}+\underset{1,2}{3} u\right)=\hat{0}_{w_{23}}+u
$$

and

$$
e-e^{\prime}=\hat{0}_{w_{23}}+\left(\hat{0}_{0_{m}^{E_{2}}}+u\right)=\hat{0}_{w_{23} 3}+\left(\odot_{m, 2}^{3}+\underset{1,3}{+} u\right)=\hat{0}_{w_{23}}+u
$$

and therefore

$$
\hat{0}_{w_{23}}+u=\hat{0}_{w_{23}}+u \text {. }
$$

\subsection{Second case: two elements that have two lower faces in common}

What happens if $e$ and $e^{\prime}$ have only two of the lower faces in common? Then only two of the three subtractions are defined. There are three cases to consider, each of which arises later.

If $e$ and $e^{\prime}$ have the same Front and Right face. Since $e$ and $e^{\prime}$ have the same $e_{1,3}$ and $e_{2,3}$, it follows that they have the same $e_{1}, e_{2}$ and $e_{3}$. However $e$ and $e^{\prime}$ will differ at $e_{1,2}$ and $e_{1,2}^{\prime}$, and these will differ by a core element $w_{12} \in E_{12}$ of the core of the Down face, that is

$$
e_{1,2} \frac{E_{E_{1}}}{e_{1,2}^{\prime}}=w_{12}+\underset{E_{2}}{\tilde{0}_{e_{1}}^{1,2},} \quad e_{1,2} \frac{{ }_{E_{2}}}{e_{1,2}^{\prime}}=w_{12} \underset{E_{1}}{+\tilde{0}_{e_{2}}^{1,2}}
$$


It is useful to write out the outlines of these differences
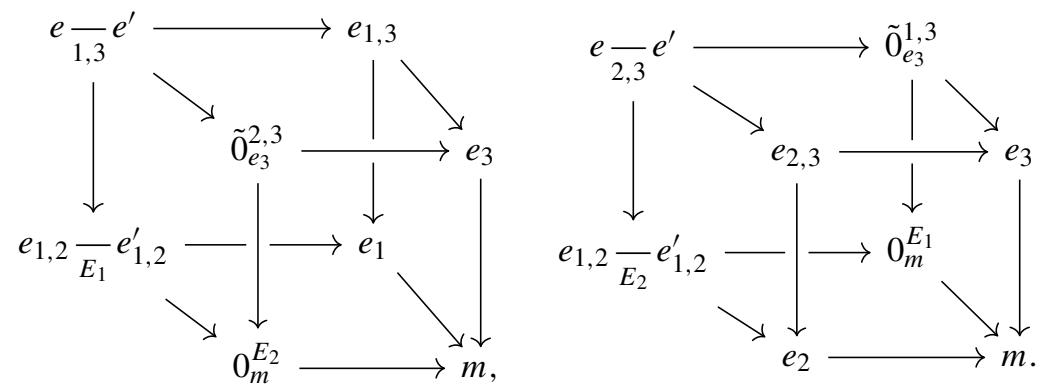

Since $e$ and $e^{\prime}$ have the same Up face, again by applying double vector bundle theory, we can write

$$
e-e_{1,3}^{\prime}=k+\hat{0}_{2,3} e_{1,3}, \quad e-e_{2,3}^{\prime}=k+\hat{0}_{e_{2,3}}
$$

where $k \in E_{23,1}$, the core of the Up face.

Also, using the morphism $q_{1,2}: E \rightarrow E_{1,2}$, we show that $q_{1,2}(k)=w_{12}$. First,

$$
q_{1,2}\left(e-e_{1,3}^{\prime}\right)=e_{1,2} \frac{-}{E_{1}} e_{1,2}^{\prime}=w_{12}+\tilde{0}_{E_{2}}^{1,2},
$$

and

$$
q_{1,2}\left(k+\hat{0}_{e_{1,3}}\right)=q_{1,2}(k) \underset{E_{2}}{+} \tilde{0}_{e_{1}}^{1,2},
$$

hence $q_{1,2}(k)=w_{12}$, where $k$ has outline

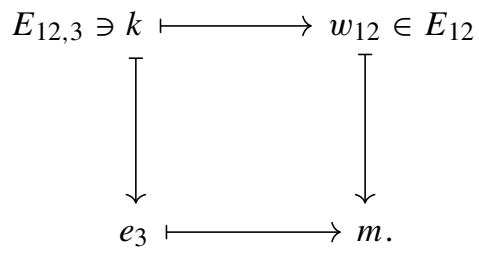

If $e$ and $e^{\prime}$ have the same Front and Down face. In this case, the elements $e_{1,3}$ and $e_{1,3}^{\prime}$ differ by a core element $w_{13}$ of $E_{13}$

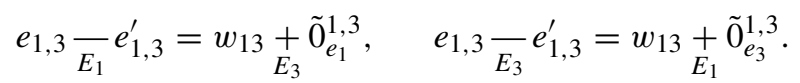

As before, we can write

$$
e \frac{-}{1,2} e^{\prime}=k+\hat{0}_{e_{1,2}, 3}, \quad e-e_{2,3}^{\prime}=k+\hat{0}_{e_{2,3}}
$$


with $k$ an element of the core of the Left face with outline

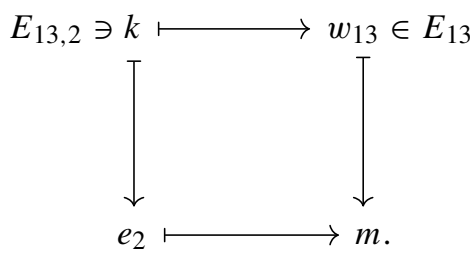

If $e$ and $e^{\prime}$ have the same Right and Down face. In this case, $e_{2,3}$ and $e_{2,3}^{\prime}$ will differ by an element $w_{23} \in E_{23}$ of the core of the Front face

$$
e_{2,3} \frac{-}{E_{2}} e_{2,3}^{\prime}=w_{23}+\tilde{0}_{E_{3}}^{2,3}, \quad e_{2,3} \frac{-e_{E_{3}}}{e_{2,3}^{\prime}}=w_{23} \underset{E_{2}}{+\tilde{0}_{e_{3}}^{2,3}},
$$

and as before

$$
e \frac{-}{1,2} e^{\prime}=k+\hat{0}_{e_{1,2}}, \quad e-\frac{1,3}{\prime} e^{\prime}=k_{1,2} \hat{0}_{e_{1,3}},
$$

where $k$ is an element of the core of the Back face with outline

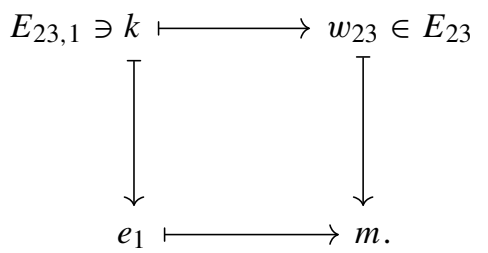

\subsection{Special case: differences between zero elements}

Using the fact that $\hat{0}$ is the zero section

$$
\hat{0}_{e_{2,3}}+\hat{0}_{e_{2,3}^{\prime}}=\hat{0}_{e_{2,3}+e_{E_{3}}^{\prime}}, \quad \hat{0}_{e_{2,3}}+\hat{0}_{e_{2,3}^{\prime}}=\hat{0}_{e_{2,3}+e_{E_{2}}^{\prime}},
$$

and $(-1)_{1,3}^{\cdot} \hat{0}_{e_{2,3}}=\hat{0}_{f_{2,3}}$ where $f_{2,3}=\frac{E_{3}}{e_{2,3}}$.

If we have two elements $e_{2,3}$ and $e_{2,3}^{\prime}$ of $E_{2,3}$ that differ by a core element $w_{23} \in E_{23}$, then

$$
e_{2,3} \frac{E_{2}}{E_{2,3}}=w_{23}+\underset{E_{3}}{+} \tilde{0}_{e_{2}}^{2,3}, \quad e_{2,3} \frac{E_{E_{3}}}{e_{2,3}^{\prime}}=w_{23}+\underset{E_{2}}{\tilde{0}_{e_{3}}^{2,3}},
$$

and the differences we are interested in are

$$
\hat{0}_{e_{2,3}}-\hat{0}_{e_{2,3}^{\prime}}=\hat{0}_{e_{2,3}}+\hat{0}_{1,2} e_{E_{2}}^{\prime} e_{2,3}=\hat{0}_{e_{2,3}-e_{E_{2}} e_{2,3}^{\prime}}=\hat{0}_{w_{23}+\tilde{0}_{E_{3}}^{2,3}}=\hat{0}_{w_{23}}+\hat{0}_{e_{2}},
$$


and

$$
\hat{0}_{e_{2,3}}-\hat{0}_{e_{2,3}^{\prime}}=\hat{0}_{e_{2,3}}+\hat{0}_{1,3} e_{E_{3}}^{\prime} e_{2,3}=\hat{0}_{e_{2,3}-e_{E_{3}}^{\prime}}=\hat{0}_{w_{23}+\tilde{0}_{E_{2}}^{2,3}}=\hat{0}_{w_{23}}+\hat{0}_{e_{3,2}} \text {. }
$$

There are similar formulas in the other two cases.

\subsection{Summary of Sect. 3}

- Given two elements $e, e^{\prime} \in E$ with the same outline, then they differ by a unique ultracore element $u \in E_{123}$ :

$$
e-e^{\prime} \triangleright u
$$

- Given two elements $e, e^{\prime} \in E$ with two lower faces in common, then their difference

$$
e-e^{\prime} \triangleright k
$$

defines a $k$ in one of the core double vector bundles:

- if $e, e^{\prime}$ have the same Right and Down face, then $k \in E_{23,1}$,

- if $e, e^{\prime}$ have the same Front and Down face, then $k \in E_{13,2}$,

- if $e, e^{\prime}$ have the same Front and Right face, then $k \in E_{12,3}$.

\section{Proof of the warp theorem}

\subsection{Notation}

In this section we prove Theorem 1. We will use the notation of (29). We further simplify the notation for elements of the lower faces and edges, as follows

$$
\begin{aligned}
& X(m):=e_{1}, \quad Y(m):=e_{2}, \quad Z(m):=e_{3}, \\
& Z_{1}(X(m)):=e_{1,3}, \quad X_{3}(Z(m)):=e_{1,3}^{\prime}, \quad Z_{2}(Y(m)):=e_{2,3}, \\
& Y_{3}(Z(m)):=e_{2,3}^{\prime}, \quad Y_{1}(X(m)):=e_{1,2}, \quad X_{2}(Y(m)):=e_{1,2}^{\prime} \text {. }
\end{aligned}
$$

The outlines of the elements in (29) are now written as follows
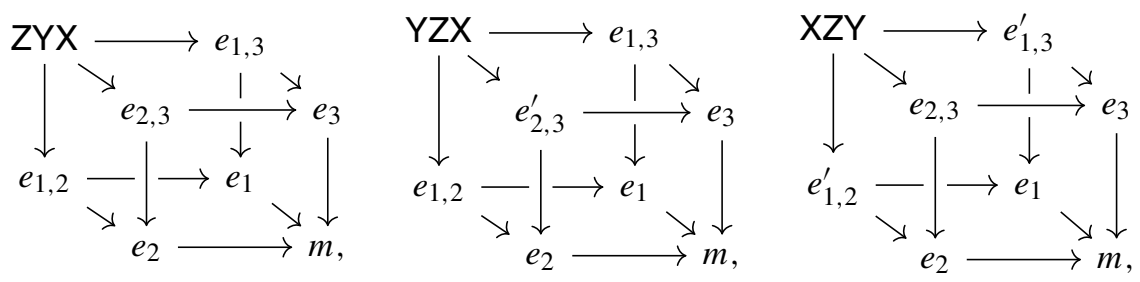

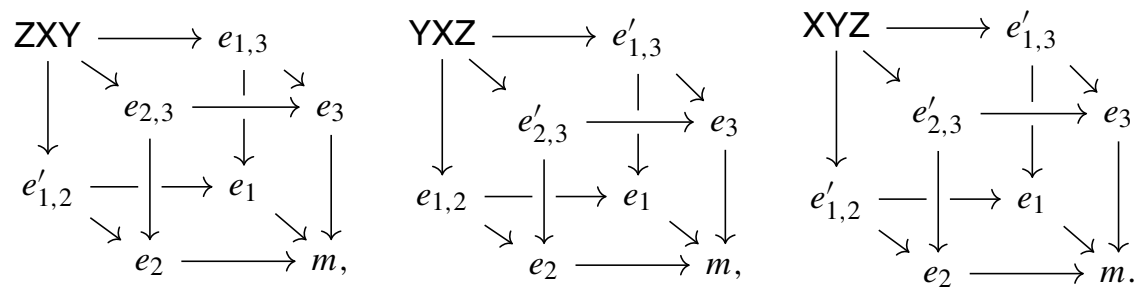

We will need the following relations for the core elements of the lower faces in detailed form.

$$
\begin{aligned}
& e_{2,3} \frac{E_{E_{2}}}{e_{2,3}^{\prime}}=\tilde{0}_{e_{2}}^{2,3} \underset{E_{3}}{+,} w_{23}, \quad e_{2,3} \frac{-}{E_{3}} e_{2,3}^{\prime}=\tilde{0}_{e_{3}}^{2,3} \underset{E_{2}}{+} w_{23} \text {, } \\
& e_{1,3}^{\prime} \frac{-}{E_{1}} e_{1,3}=\tilde{0}_{e_{1}}^{1,3} \underset{E_{3}}{+} w_{13}, \quad e_{1,3}^{\prime} \frac{{ }_{E_{3}}}{e_{1,3}}=\tilde{0}_{e_{3}}^{1,3} \underset{E_{1}}{+} w_{13} \text {, } \\
& e_{1,2} \frac{E_{1}}{E_{1,2}}=\tilde{0}_{e_{1}}^{1,2} \underset{E_{2}}{+} w_{12}, \quad e_{1,2} \frac{{ }_{E_{2}}}{e_{1,2}^{\prime}}=\tilde{0}_{e_{2}}^{1,2} \underset{E_{1}}{+} w_{12} \text {, }
\end{aligned}
$$

where $w_{23} \in E_{23}, w_{13} \in E_{13}$ and $w_{12} \in E_{12}$.

For the zeros of these $w$ elements, the diagrams are
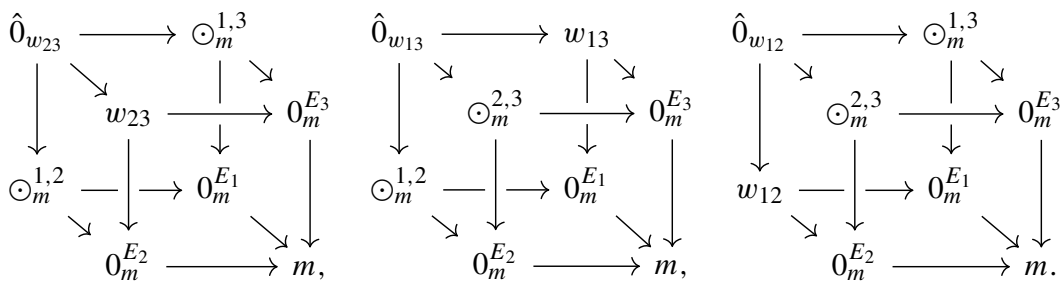

\subsection{Core and ultracore elements arising from the grid}

We collect here for reference the definitions and outlines of the core and ultracore elements arising from the grid.

- $\lambda_{1}, k_{1}$ and $u_{1}$. The elements ZYX and YZX have the same Right and Back faces, and so their differences define an element $\lambda_{1} \in E_{23,1}$ with outline

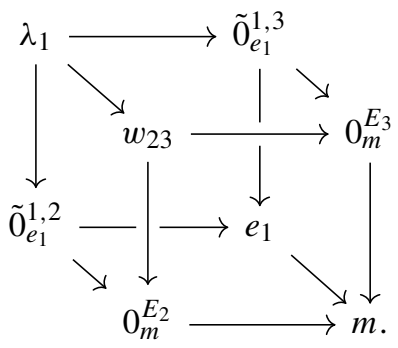


Using (40) the defining equations are

$$
Z Y X \frac{Y Z X}{-1,2}=\hat{0}_{e_{1,2}}+\lambda_{1,3}, \quad Z Y X-Y Z X=\hat{0}_{e_{1,3}}+\lambda_{1,2}
$$

If we look at $X Z Y$ and $X Y Z$, we see that they also have two faces in common, and their differences define a $k_{1} \in E_{23,1}$, with outline

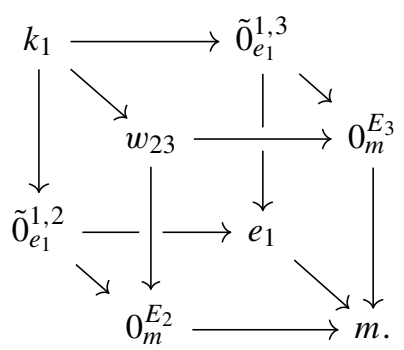

The two differences are, again using (40),

$$
\mathrm{XZY} \frac{1,2}{\mathrm{XYZ}}=\hat{0}_{e_{1,2}^{\prime}}+k_{1,3}, \quad \mathrm{XZY} \frac{\mathrm{1,3}}{\mathrm{XYZ}}=\hat{0}_{e_{1,3}^{\prime}}+k_{1,2}
$$

We see that $\lambda_{1}$ and $k_{1}$ have the same outlines so they differ by an ultracore element $u_{1} \in E_{123}$,

$$
\begin{aligned}
& \lambda_{1} \frac{-1,3}{1,3}=\hat{0}_{e_{1}}+u_{2,3}, \\
& \lambda_{1} \frac{-}{1,2} k_{1}=\hat{0}_{e_{1}}+u_{1}, \\
& \lambda_{1} \frac{-}{2,3} k_{1}=\hat{0}_{w_{23}}+u_{1,3}=\hat{0}_{w_{23}}+u_{1,2} .
\end{aligned}
$$

There are four ways of describing the warp

$$
\begin{aligned}
& \left(Z Y X \frac{-Y Z X)}{-1,3}(X Z Y \underset{1,2}{-} X Y Z)=\hat{0}_{e_{1}}+\left(\hat{0}_{w_{12}}+u_{1,3 / 2,3}^{+} u_{1}\right)\right. \\
& \left(Z Y X \frac{-Y Z X)}{2,3}\left(X Z Y \frac{1,2}{-X Y Z}\right)=\left(\hat{0}_{w_{12}}+\hat{0}_{w_{23}}\right)+\left(\hat{0}_{e_{2}}+u_{1,3}\right)\right. \\
& (Z Y X \underset{1,3}{-} \mathrm{YZX}) \underset{1,2}{-}(\mathrm{XZY} \underset{1,3}{-} \mathrm{XYZ})=\hat{0}_{e_{1}}+\left(\hat{0}_{-w_{13}} \underset{1,2 / 2,3}{+} u_{1}\right) \\
& \left(Z Y X \frac{-Y Z X)}{2,3}\left(X Z Y \frac{1,3}{-} X Y Z\right)=\left(\hat{0}_{-w_{13}}+\hat{0}_{1,2} w_{23}\right)+\left(\hat{0}_{e_{3}}+u_{1,2}\right)\right.
\end{aligned}
$$

Here we write $\hat{0}_{w_{12}}+u_{1,3 / 2,3}$ to denote $\hat{0}_{w_{12}}+u_{1,3}=\hat{0}_{w_{12}}+u_{1,3}$. 
- $\lambda_{2}, k_{2}$ and $u_{2}$. The same procedure can be applied to XZY and ZXY; they have the same Front and Down faces, so their differences will define an element $\lambda_{2} \in E_{13,2}$

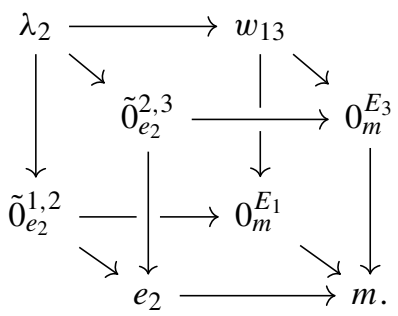

The corresponding equations, using (39), are

$$
X Z Y \frac{Z X Y}{1,2}=\hat{0}_{e_{1,2}^{\prime}}+\lambda_{2,3}, \quad X Z Y \frac{Z X Y}{2,3}=\hat{0}_{e_{2,3}}+\lambda_{1,2}
$$

If we look at $Y X Z$ and $Y Z X$, their differences define a $k_{2} \in E_{13,2}$, with outline

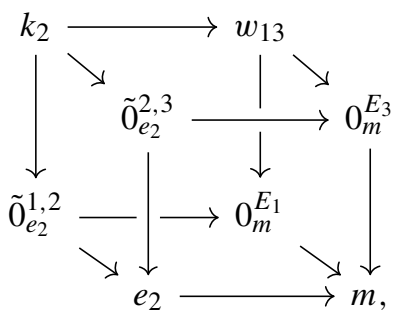

and the differences defined are, due to (39),

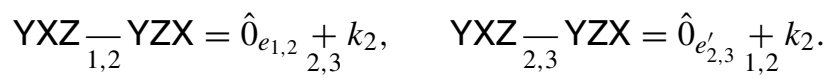

Since $\lambda_{2}$ and $k_{2}$ have the same outlines, they differ by an ultracore element $u_{2} \in E_{123}$,

$$
\begin{aligned}
& \lambda_{2 \frac{1,3}{1,3} k_{2}}=\hat{0}_{w_{13} \underset{1,2 / 2,3}{+} u_{2},} \\
& \lambda_{2 \frac{1,2}{1,2} k_{2}}=\hat{0}_{e_{2}}+u_{1,3}, \\
& \lambda_{2} \frac{-}{2,3} k_{2}=\hat{0}_{e_{2}}+u_{1,3} .
\end{aligned}
$$

Again there are four ways of describing the warp

$$
\begin{aligned}
& (X Z Y \underset{1,2}{-Z X Y}) \underset{2,3}{-}\left(Y X Z \frac{Y Z X)}{1,2}=\hat{0}_{e_{2}}+\left(\hat{0}_{-w_{12}} \underset{1,3 / 2,3}{+} u_{2}\right),\right.
\end{aligned}
$$

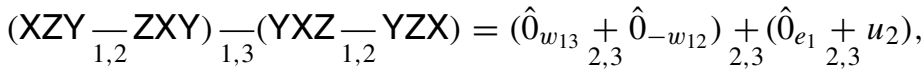




$$
\begin{aligned}
& \left(X Z Y \frac{2,3}{Z X Y}\right) \underset{1,2}{-}\left(Y X Z \frac{2,3}{Y Z X}\right)=\hat{0}_{e_{2}}+\left(\hat{0}_{w_{23}} \underset{1,2 / 1,3}{+} u_{2}\right),
\end{aligned}
$$

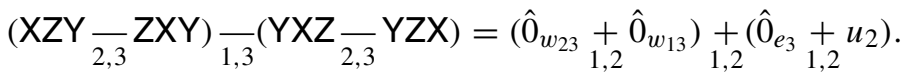

- $\lambda_{3}, k_{3}$ and $u_{3}$. Likewise $\mathrm{YXZ}$ and $\mathrm{XYZ}$ define $\lambda_{3} \in E_{12,3}$ with outline

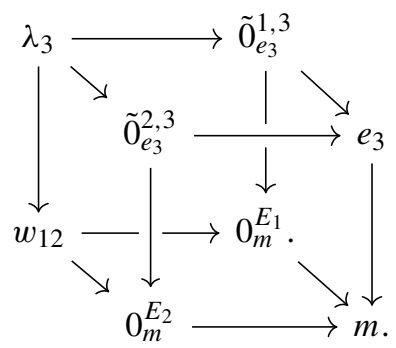

The corresponding relations are, due to (38),

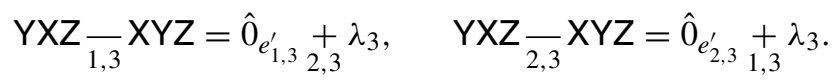

Likewise $Z Y X$ and $Z X Y$ define a $k_{3} \in E_{12,3}$ with outline

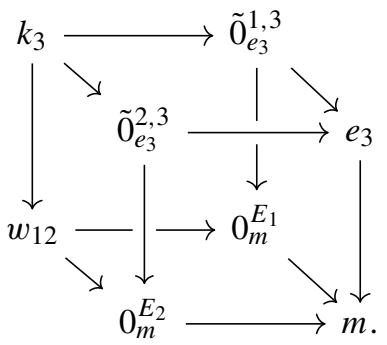

The differences defined are, due to (38),

$$
Z Y X \frac{Z X Y}{1,3}=\hat{0}_{e_{1,3}}+k_{2,3}, \quad Z Y X \frac{Z X Y}{2,3}=\hat{0}_{e_{2,3}}+k_{1,3}
$$

The ultracore element $u_{3} \in E_{123}$ defined by $\lambda_{3}$ and $k_{3}$ satisfies

$$
\begin{aligned}
& \lambda_{3} \frac{-3,3}{1,3}=\hat{0}_{e_{3}}+u_{3,2}, \\
& \lambda_{3} \frac{-}{1,2} k_{3}=\hat{0}_{w_{12}}+u_{1,3 / 2,3}^{+} u_{3}, \\
& \lambda_{3} \frac{-}{2,3} k_{3}=\hat{0}_{e_{3}}+u_{3,2} .
\end{aligned}
$$


The four relations in this case are

$$
\begin{aligned}
& \left.(\mathrm{YXZ} \underset{1,3}{-\mathrm{XYZ}}) \frac{-\mathrm{ZYX}}{2,3} \underset{1,3}{-\mathrm{ZXY}}\right)=\hat{0}_{e_{3}}+\left(\hat{0}_{w_{13}} \underset{1,2 / 2,3}{+} u_{3}\right),
\end{aligned}
$$

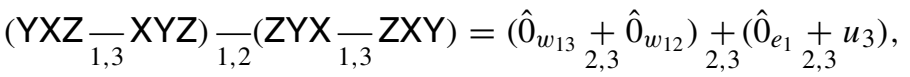

$$
\begin{aligned}
& \left(Y X Z \frac{2,3}{-X Y Z}\right) \underset{1,3}{-}\left(Z Y X \frac{Z X Y)}{2,3} \hat{0}_{e_{3}}+\left(\hat{0}_{-w_{23}} \underset{1,2 / 1,3}{+} u_{3}\right),\right.
\end{aligned}
$$

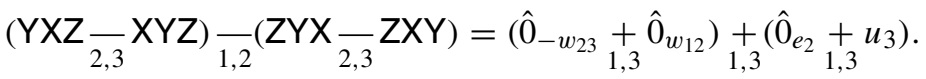

\subsection{Proof of the warp theorem}

We will show that $u_{1}+u_{2}+u_{3}=\odot_{m}^{3}$ by showing that $u_{1}=-u_{2}-u_{3}$. There are four steps.

Step 1. Rewrite (49b),

$$
(Z Y X \underset{1,2}{-} Y Z X) \underset{2,3}{-}(X Z Y \underset{1,2}{-} X Y Z) \text { as }(Z Y X \underset{2,3}{-} X Z Y) \underset{1,2}{-}\left(Y Z X \frac{2,3}{-} X Y Z\right)
$$

using the double vector bundle interchange law in the Left face. We know from (49b) that the ultracore element defined by the first expression is $u_{1}$, therefore, the ultracore element of the latter expression will also be $u_{1}$. We will show that the second expression has $-u_{2}-u_{3}$ as its ultracore element, and this will show that $u_{1}=-u_{2}-u_{3}$.

Step 2. First, write $Z Y X \frac{-}{2,3} X Y Y$ as

$$
Z Y X \frac{-2,3}{2 Z Y}=\left(Z Y X \frac{-Z X Y)}{2,3}\left(X Z Y \frac{2,3}{2,3} Z Y\right)\right.
$$

where we have that

$$
Z Y X \frac{Z}{2,3} \mathrm{ZXY}=\hat{0}_{e_{2,3}}+k_{1,3}, \quad \mathrm{XZY} \frac{\mathrm{Z}, \mathrm{ZY}}{2,3}=\hat{0}_{e_{2,3}}+\lambda_{1,2}
$$

Step 3. Similarly, write $Y Z X \frac{2,3}{-} X Y Z$ as

$$
Y Z X \underset{2,3}{-} X Y Z=(Y X Z \underset{2,3}{-} X Y Z) \underset{2,3}{-}(Y X Z \underset{2,3}{-} Y Z X)
$$

and we have

$$
\mathrm{YXZ} \frac{\mathrm{2,3}}{\mathrm{XYZ}}=\hat{0}_{e_{2,3}^{\prime}}+\lambda_{1,3}, \quad \mathrm{YXZ} \frac{\mathrm{2,3}}{\mathrm{YZX}}=\hat{0}_{e_{2,3}^{\prime}}+k_{1,2}
$$

Step 4. Since our convention is that $\lambda_{3}-k_{3}$ defines $u_{3}$, it follows that $k_{3}-\lambda_{3}$ defines $-u_{3}$. It is essential to follow these conventions. 
We are finally able to complete the proof of Theorem 1 . First, using operations in $E \rightarrow E_{2,3}$, we have

$$
\begin{aligned}
& (Z Y X \underset{2,3}{-} X Z Y) \underset{1,2}{-}\left(Y Z X \frac{-}{2,3} X Y Z\right)
\end{aligned}
$$

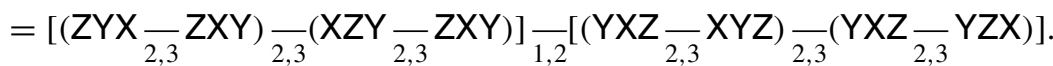

Now, using (54) and (55), this is equal to

$$
\left[\left(\hat{0}_{e_{2,3}}+k_{1,3}\right) \underset{2,3}{-}\left(\hat{0}_{e_{2,3}}+\lambda_{1,2}\right)\right] \underset{1,2}{-}\left[\left(\hat{0}_{e_{2,3}^{\prime}}+\lambda_{1,3}\right) \underset{2,3}{-}\left(\hat{0}_{e_{2,3}^{\prime}}+k_{1,2}\right)\right] .
$$

Applying the interchange law in the Left face to the outer operations, this becomes

$$
\left[\left(\hat{0}_{e_{2,3}}+k_{1,3}\right) \underset{1,2}{-}\left(\hat{0}_{e_{2,3}^{\prime}}+\lambda_{1,3}\right)\right] \underset{2,3}{-}\left[\left(\hat{0}_{e_{2,3}}+\lambda_{1,2}\right) \underset{1,2}{-}\left(\hat{0}_{e_{2,3}^{\prime}}+k_{1,2}\right)\right] .
$$

Applying the interchange law in the Back face, in each [ ], we have

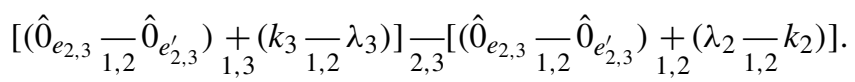

Now apply (42) to the first term in each [ ]. Then use (52b) and (50b), remembering that $k_{3}-\lambda_{3}$ defines $-u_{3}$. This gives

$$
\left[\left(\hat{0}_{w_{23}}+\hat{0}_{e_{2}}\right)+\left(\hat{0}_{w_{12}}-u_{3,3}\right)\right] \underset{2,3}{-}\left[\left(\hat{0}_{w_{23}}+\hat{0}_{1,3}\right)+\left(\hat{0}_{e_{2}}+u_{2}\right)\right] .
$$

Now apply the interchange law in the Back face to the second [ ] :

$$
\left[\hat{0}_{w_{23}}+\left(\hat{0}_{e_{2}}+\hat{0}_{1,3} w_{12}-u_{3,3}\right)\right] \frac{2,3}{-}\left[\left(\hat{0}_{w_{23}}+u_{1,2}\right)+\left(\hat{0}_{e_{2}}+\hat{0}_{e_{2}}\right)\right] .
$$

Using (37) in the first ( ) of the second [ ], and noting that the zeros in the last ( ) are zeros over $E_{1,2}$, this in turn is equal to

$$
\left[\hat{0}_{w_{23}}+\left(\hat{0}_{e_{2}}+\hat{0}_{w_{12}}-u_{1,3}\right)\right] \underset{2,3}{-}\left[\left(\hat{O}_{w_{23}}+u_{2,3}\right)+\hat{0}_{e_{2}}\right]
$$

Rewrite this as :

$$
\left[\hat{0}_{w_{23}}+\left(\hat{0}_{e_{2}}+\hat{0}_{1,3} w_{12}-u_{3,3}\right)\right] \underset{2,3}{-}\left[\hat{0}_{w_{23}}+\left(\hat{0}_{e_{2}}+u_{1,3}\right)\right]
$$

note that the second [ ] is in an ordinary vector bundle. Now use the interchange law in the Up face :

$$
\left[\hat{0}_{w_{23}}-\hat{0}_{2,3} w_{23}\right]+\left[\left(\hat{0}_{e_{2}}+\hat{0}_{w_{12}}-u_{3,3}\right) \underset{2,3}{-}\left(\hat{0}_{e_{2}}+u_{1,3}\right)\right]
$$


and this is equal to

$$
\hat{0}_{w_{23}}+\left[\hat{0}_{e_{2}}-\hat{0}_{2,3}\right]+\underset{1,3}{+}\left[\left(\hat{0}_{w_{12}}-u_{1,3}\right) \frac{-}{2,3} u_{2}\right]
$$

using the facts that the zeros $\hat{0}_{w_{23}}$ in the first [ ] are zeros over $E_{2,3}$, and then the interchange law in the Up face. Likewise, using the fact that the zeros $\hat{0}_{e_{2}}$ are zeros over $E_{2,3}$, this is equal to

$$
\hat{0}_{w_{23}}+\hat{0}_{e_{2}}+\left[\left(\hat{0}_{w_{12}}-u_{2,3}\right) \frac{-3}{2,3} u_{2}\right]
$$

Finally, using an equation of the form (37), this becomes

$$
\hat{0}_{w_{23}}+\hat{0}_{e_{2}}+\left[\hat{0}_{w_{12}}-\left(u_{3,3}+u_{2,3}\right)\right]=\hat{0}_{w_{23}}+\hat{0}_{1,3}+\hat{0}_{e_{2}}+\hat{0}_{w_{12}}-\left(u_{1,3}+u_{1,3}\right),
$$

from which we obtain $-\left(u_{3}+u_{2}\right)$ as the ultracore element.

Comparing this with (49b),

$$
\left(Z Y X \frac{-Y Z X)}{2,3}\left(X Z Y \frac{1,2}{-X Y Z}\right)=\left(\hat{0}_{w_{12}}+\hat{0}_{w_{23}}\right) \underset{1,3}{+}\left(\hat{0}_{e_{2}}+u_{1,3}\right)\right.
$$

we have $u_{1}=-\left(u_{3}+u_{2}\right)$ as desired.

This completes the proof of the warp theorem.

\subsection{Summary of Sect. 4}

A grid on $E$, for $m \in M$, defines six elements, ZYX, YZX, XZY, ZXY, YXZ, XYZ. These elements define the following core and ultracore elements:

$$
\begin{array}{lll}
Z Y X-Y Z X \triangleright \lambda_{1} \in E_{23,1}, & \mathrm{XZY}-\mathrm{XYZ} \triangleright k_{1} \in E_{23,1}, & \lambda_{1}-k_{1} \triangleright u_{1}, \\
\mathrm{XZY}-\mathrm{ZXY} \triangleright \lambda_{2} \in E_{13,2}, & \mathrm{YXZ}-\mathrm{YZX} k_{2} \in E_{13,2}, & \lambda_{2}-k_{2} \triangleright u_{2}, \\
\mathrm{YXZ}-\mathrm{XYZ} \triangleright \lambda_{3} \in E_{12,3}, & \mathrm{ZYX}-\mathrm{XXY} \triangleright k_{3} \in E_{12,3}, & \lambda_{3}-k_{3} \triangleright u_{3} .
\end{array}
$$

In the final step of the proof we showed that

$$
\begin{aligned}
& (Z Y X-Y Z X)-(X Z Y-X Y Z) \triangleright u_{1}, \\
& {[(Z Y X-Z X Y)-(X Z Y-Z X Y)]-[(Y X Z-X Y Z)-(Y X Z-Y Z X)] \triangleright-\left(u_{2}+u_{3}\right),}
\end{aligned}
$$

and since the left-hand sides are equal, it follows that $u_{1}=-u_{2}-u_{3}$.

Remark 1 The strategy of this proof deserves some commentary.

What should the warp of a grid on a triple vector bundle be? Or, in other words, why are we interested in the ultrawarps of a grid of a triple vector bundle?

The warp of a grid in the double case is a section of the core vector bundle, and measures the non-commutativity of the two routes defined by the grid. 
So far, we have seen that all operations on a triple vector bundle are iterations of operations defined in double vector bundles. The ultracore, for example, is the core of the core double vector bundles.

For these reasons, we would want the warp of a grid in the triple case to be a section of the ultracore vector bundle, and to measure the non-commutativity of routes defined by the grid.

Pick an upper face of $E$, for example the Up face. If we compare the two routes defined by the grid in this face, then we obtain an element of the (U-D) core double vector bundle, which we denoted by $\lambda_{3}$. Similarly for the other upper faces, the non-commutativity of the corresponding routes defines $\lambda_{1}$ and $\lambda_{2}$. The three $\lambda$ 's are elements of different spaces; therefore, if we tried to compare them, or indeed perform any sort of operation with them (such as adding them or subtracting them), we would see that such an operation could be algebraically possible but would not be geometrically meaningful.

The same applies for the three $k_{i}$ defined by the comparison of the routes for the lower faces.

The $\lambda_{i}$ 's and the corresponding $k_{i}$ 's, however, are elements of the same spaces, and so comparing them is a possibility, and indeed the only sensible operation. And by comparing them, we measure the non-commutativity of four routes, instead of two.

This can be done for the three pairs of $\lambda_{i}$ and $k_{i}$, and so we obtain the three ultrawarps.

So what does the warp theorem tell us?

Each ultrawarp measures the non-commutativity of four routes. In total, a grid on a triple vector bundle provides six different routes from $M$ to $E$. The sum of the three ultrawarps takes into account each route twice, once with a positive and once with a negative sign. (Compare the description of curvature identities in terms of paths around oriented faces in cubes and hypercubes given by [20, Chap. 15].) The warp theorem tells us that these add up to zero, a result that seems reasonable. The different vector bundle structures over which the operations take place, however, are the main obstacle here-as soon as one realizes that simple operations like addition and subtraction in the triple vector bundle setting are no longer simple. Further analysis of grids in triple vector bundles is given in [5].

\section{The triple vector bundle $T^{2} A$ and connections in $A$}

In Example 1 of the Introduction we started with a vector bundle $A \rightarrow M$ and a connection in $A$, and we saw that the warp of the grid (9) is $\nabla_{Z} \mu$, the covariant derivative of $\mu$ with respect to $Z$. In this section, starting with a vector bundle $A \rightarrow M$ and a connection in $A$, we will build a grid on the triple vector bundle $T^{2} A$. We now apply the warp theorem to this grid, and we thereby obtain a new proof of the equivalence of the definition of curvature in terms of horizontal lifts and the definition in terms of covariant derivatives (see Theorem 2). 
For a vector bundle $(A, q, M)$ there is a triple vector bundle structure on $T^{2} A$ as shown in (56).

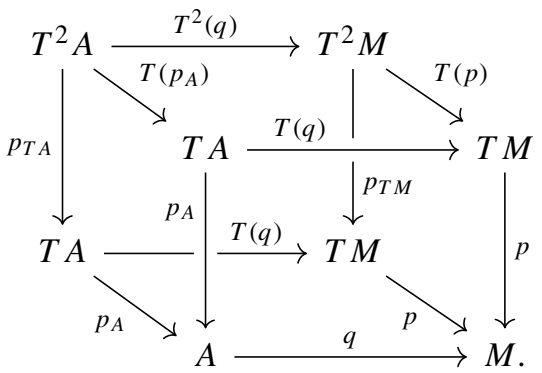

Here the Down face is the usual double vector bundle $T A$ and the Up face is the tangent prolongation of this; that is, it is obtained by applying the tangent functor to each structure in $T A$. Each vertical vector bundle in (56) is a standard tangent bundle.

In the first subsection we describe in detail the core double vector bundles of $T^{2} A$. In the second subsection we investigate $J_{A}: T^{2} A \rightarrow T^{2} A$, the canonical involution of the manifold $A$, as a morphism of triple vector bundles. In the third subsection we build the aforementioned grid on $T^{2} A$, and in the two remaining subsections we calculate the three ultrawarps induced by this grid.

\subsection{The core double vector bundles of $T^{2} A$}

The three core double vector bundles of (56) are shown in (57), in the usual order (B-F), (L-R), and (U-D), and arranged as in (14).

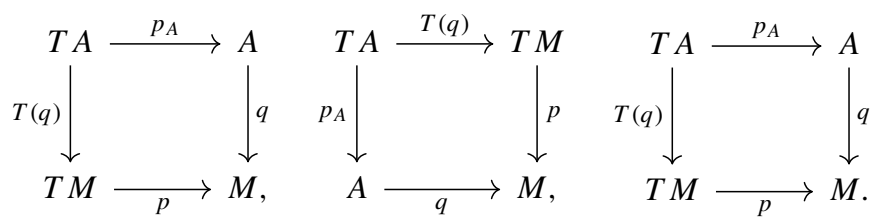

These core double vector bundles are the same as abstract double vector bundles but are embedded differently in $T^{2} A$.

An element $\xi \in T A$ determines core elements of the Back, Left and Up faces. We denote these by $\bar{\xi}^{B}, \bar{\xi}^{L}$ and $\bar{\xi}^{U}$, respectively. Since they are elements of $T(T A)$, they can be represented as tangent vectors to curves in $T A$.

The Back face is the tangent double vector bundle for the tangent prolongation bundle $T(q): T A \rightarrow T M$. For the core of the Back face we have

$$
\bar{\xi}^{B}=\left.\frac{d}{d t}\left(t_{T M}^{.} \xi\right)\right|_{t=0},
$$

where $t_{T M}^{.} \xi$ denotes scalar multiplication in $T(q): T A \rightarrow T M$. 
The Left face is the double tangent bundle of the manifold $A$. Given $\xi \in T A$ the core element is

$$
\bar{\xi}^{L}=\left.\frac{d}{d t}(t \xi)\right|_{t=0}
$$

where the scalar multiplication is in the usual tangent bundle $T A \rightarrow A$.

For the core of the Up face, first write $\xi=\left.\frac{d}{d t} a_{t}\right|_{t=0}$ where $a_{t}$ is a curve in $A$. Write $\overline{a_{t}} \in T A$ for the core element corresponding to $a_{t}$. Then

$$
\bar{\xi}^{U}=\left.\frac{d}{d t}\left(\overline{a_{t}}\right)\right|_{t=0} .
$$

\subsection{The canonical involution on $T^{2} A$}

The canonical involution $J_{A}: T^{2} A \rightarrow T^{2} A$ for the manifold $A$ is an isomorphism from the double vector bundle $T^{2} A$ to its flip. In what follows we will need to use it as a map of triple vector bundles.

Proposition 3 The map $J_{A}$ is an isomorphism of the triple vector bundles shown in (61).
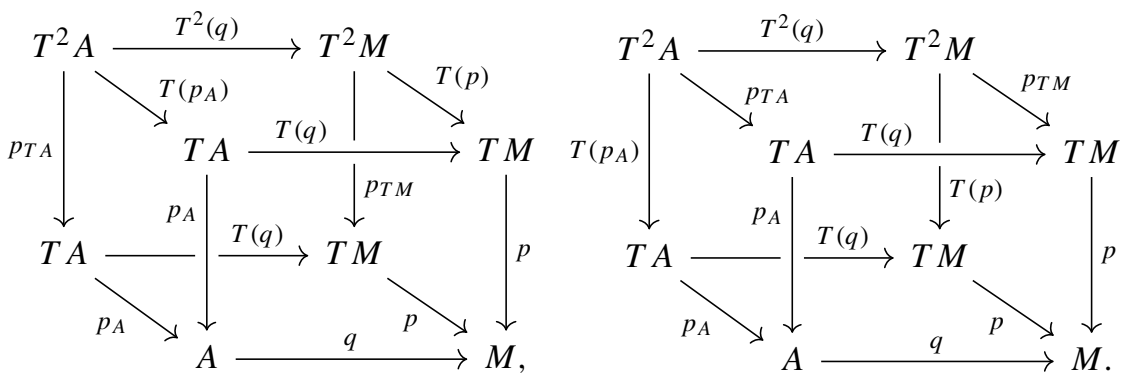

In (61) the Left faces are the double tangent bundles of the manifold $A$ and $J_{A}$ maps the Left face of the domain to its flip. It interchanges the Up and Back faces. The Right faces are the double tangent bundles of $M$ and $J_{A}$ induces $J_{M}: T^{2} M \rightarrow T^{2} M$ which maps the Right face of the domain to its flip. The Front and Down faces are interchanged.

The proof of Proposition 3 relies on the following two lemmas. The first is the naturality property of the canonical involution.

Lemma 2 Let $M$ and $N$ be smooth manifolds, and $F: M \rightarrow N$ a smooth map. Then $T^{2}(F) \circ J_{M}=J_{N} \circ T^{2}(F)$, where $T^{2}(F)=T(T(F))$ is the tangent of the tangent map $T(F)$.

Lemma 3 Given $\Phi_{1}, \Phi_{2} \in T^{2} A$, over the same $\xi \in T^{2} M$, we have

$$
J_{A}\left(\Phi_{1} \underset{T^{2}(q)}{+} \Phi_{2}\right)=J_{A}\left(\Phi_{1}\right) \underset{T^{2}(q)}{+} J_{A}\left(\Phi_{2}\right) .
$$


Consider now the maps which $J_{A}$ induces on the cores.

Take an element $\xi \in T A$ in the core of the Back face. Regarded as an element of $T^{2} A$ this is $\bar{\xi}^{B}$, with outline shown on the left of (62).
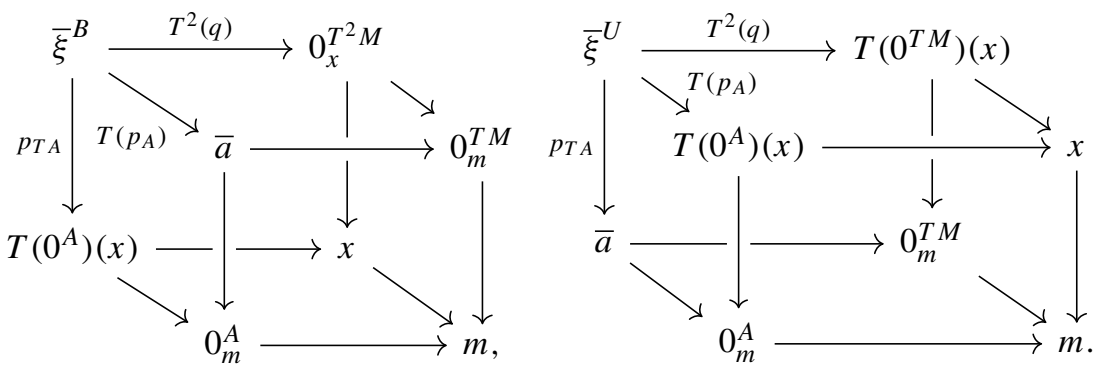

It follows from (58) and (60) that

$$
J_{A}\left(\bar{\xi}^{B}\right)=\bar{\xi}^{U} \text { and } J_{A}\left(\bar{\xi}^{U}\right)=\bar{\xi}^{B},
$$

since $J_{A}^{2}$ is the identity.

Since the Left faces in (61) are the double tangent bundle $T^{2} A$, the map on the cores of the Left faces is the identity and so

$$
J_{A}\left(\bar{\xi}^{L}\right)=\bar{\xi}^{L}
$$

\subsection{Grids on $T^{2} A$}

Now consider a connection $\nabla$ in $A$. Example 1 gave a construction of a grid in $T A$ for which the warp is $\nabla_{X} \mu$. We now extend this idea to define a grid in $T^{2} A$.

Let $X, Z \in \mathfrak{X}(M)$, and $\mu \in \Gamma A$. Define the following three linear double sections:

- From Front to Back face: $\left(T\left(X^{H}\right) ; X^{H}, T(X) ; X\right)$.

- From Right to Left face: $\left(T^{2}(\mu) ; T(\mu), T(\mu) ; \mu\right)$.

- From Down to Up face: $\left(\widetilde{Z}^{A} ; \widetilde{Z}, Z^{H} ; Z\right)$.

Here $\widetilde{Z}=J_{M} \circ T(Z)$ is the complete (or tangent) lift of $Z$ to a vector field on $T M$. Likewise $\widetilde{Z^{H}}{ }^{A}$ is the complete lift of $Z^{H} \in \mathfrak{X}(A)$ to a vector field on $T A$. The grid is 
shown in (65).

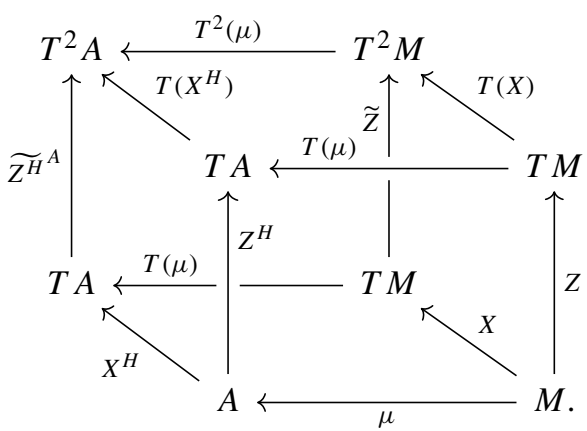

The core morphisms of the linear double sections will be needed later:

- For $\left(T\left(X^{H}\right) ; X^{H}, T(X) ; X\right)$ the core morphism is $\left(X^{H}, X\right)$.

- For $\left(T^{2}(\mu) ; T(\mu), T(\mu) ; \mu\right)$ the core morphism is $(T(\mu), \mu)$.

- For $\left(\widetilde{Z^{H}}{ }^{A} ; \widetilde{Z}, Z^{H} ; Z\right)$ the core morphism is $\left(Z^{H}, Z\right)$.

The first two cases are instances of the general fact that given a morphism $(\varphi, f)$ of vector bundles, the core morphism of the double vector bundle map $(T(\varphi) ; \varphi, T(f) ; f)$ is $(\varphi, f)$.

To calculate the core morphism of $\left(\widetilde{Z^{H}}{ }^{A} ; \widetilde{Z}, Z^{H} ; Z\right)$, focus on (66). At this point we investigate this linear double section further; it is a double vector bundle morphism from the Down face to the Up face of $T^{2} A$. Note that (66) is not a triple vector bundle.

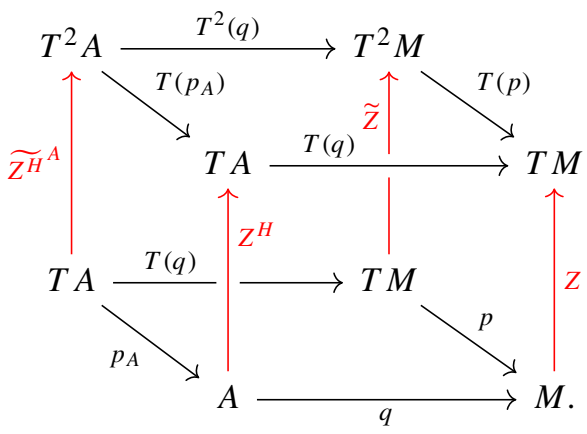

Take an element $a \in A$. As an element of the core of the Down face of (65) it is $\bar{a}=\left.\frac{d}{d t} t a\right|_{t=0} \in T A$. Using the fact that $(\widetilde{Z}, Z)$ is a vector bundle map, we have that $\widetilde{Z}\left(0_{m}^{T M}\right)=T\left(0^{T M}\right)(Z(m))$.

Similarly, using the fact that $\left(Z^{H}, Z\right)$ is a vector bundle map, we have that $Z^{H}\left(0_{m}^{A}\right)=T\left(0^{A}\right)(Z(m))$. Finally,

$$
\begin{aligned}
{\widetilde{Z^{H}}}^{A}(\bar{a}) & =J_{A}\left(T\left(Z^{H}\right)(\bar{a})\right)=J_{A}\left(\left.\frac{d}{d t} Z^{H}(t a)\right|_{t=0}\right) \\
& =J_{A}\left(\left.\frac{d}{d t} t Z^{H}(a)\right|_{t=0}\right)=J_{A}\left({\overline{Z^{H}(a)}}^{B}\right) .
\end{aligned}
$$


Table 1 Warps of grids of each face of $T^{2} A$

\begin{tabular}{ll}
\hline $\mathrm{w}_{\text {back }}$ & $\mathrm{w}_{\text {front }}$ \\
\hline$\widetilde{Z}^{A} \circ T(\mu)-T^{2}(\mu) \circ \widetilde{Z} \triangleright-T\left(\nabla_{Z} \mu\right)$ & $Z^{H} \circ \mu-T(\mu) \circ Z \triangleright-\nabla_{Z} \mu$ \\
\hline $\mathrm{w}_{\text {left }}$ & $\mathrm{w}_{\text {right }}$ \\
\hline$T\left(X^{H}\right) \circ Z^{H}-{\widetilde{Z^{H}}}^{A} \circ X^{H} \triangleright\left[Z^{H}, X^{H}\right]$ & $T(X) \circ Z-\widetilde{Z} \circ X \triangleright[Z, X]$ \\
\hline $\mathrm{w}_{\text {up }}$ & $\mathrm{w}_{\text {down }}$ \\
\hline$T^{2}(\mu) \circ T(X)-T\left(X^{H}\right) \circ T(\mu) \triangleright T\left(\nabla_{X} \mu\right)$ & $T(\mu) \circ X-X^{H} \circ \mu \triangleright \nabla_{X} \mu$ \\
\hline
\end{tabular}

Note the following. Initially, $\bar{a} \in T A$ is in the core of the Down face of $T^{2} A$. The canonical involution $J_{A}$ maps the Down face to the Front face (see (61)). Therefore, in $T\left(Z^{H}\right)(\bar{a}), \bar{a}$ is now an element of the core of the Front face. The maps $\left(T\left(Z^{H}\right) ; Z^{H}, T(Z) ; Z\right)$ form a double vector bundle morphism from the Front to the Back face of (56), with core morphism $\left(Z^{H}, Z\right)$ as usual. Therefore, $T\left(Z^{H}\right)(\bar{a})={\overline{Z^{H}(a)}}^{B}$ is now in the core of the Back face. And by (63), it follows that $J_{A}\left({\overline{Z^{H}(a)}}^{B}\right)={\overline{Z^{H}(a)}}^{U}$.

This completes the proof that the core morphism of $\left(\widetilde{Z^{H}}{ }^{A} ; \widetilde{Z}, Z^{H} ; Z\right)$ is $\left(Z^{H}, Z\right)$.

\subsection{The six warps of grid (65)}

The first step in applying the warp theorem to the grid (65) on $T^{2} A$ was to calculate the core morphisms of the linear double sections of the grid; we have done this in the previous subsection. Now, for each face of $T^{2} A$, we find the warp of the grid defined by (65). We present the six warps in Table 1, using the $\triangleright$ notation introduced directly after Eq. (4), on page 3.

The three warps $\mathrm{w}_{\text {front }}, \mathrm{w}_{\text {right }}$, and $\mathrm{w}_{\text {down }}$ of the lower faces follow directly from Example 1 and Eq. (5). The warps of the upper faces are a bit trickier.

We begin with the warp of the Up face. For this, we need the following proposition which states that the warp of the tangent of a grid is the tangent of the warp of the grid.

Proposition 4 Let $(\xi, X)$ and $(\eta, Y)$ be a grid on a double vector bundle $D$ with warp $\mathrm{w}(\xi, \eta) \in \Gamma C$. Then $(T(\xi), T(X))$ and $(T(\eta), T(Y))$ form a grid on the double vector bundle $T D$ in (67) below and the warp of the tangent grid $(T(\xi), T(X)),(T(\eta), T(Y))$ is $T(\mathrm{w}(\xi, \eta)) \in \Gamma_{T M}(T C)$. 

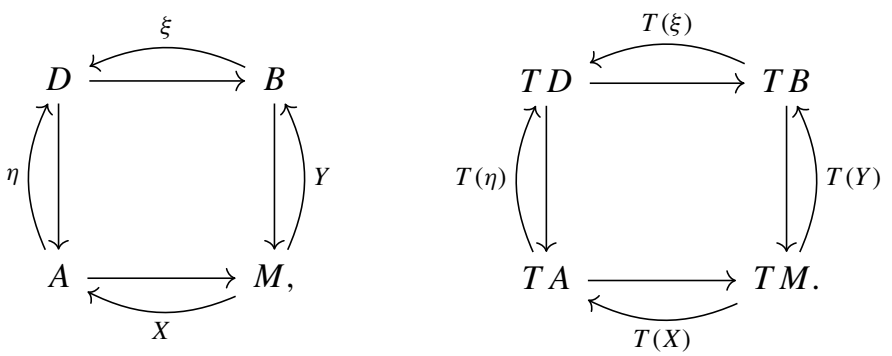

Proof We leave the verification that $T D$ is a double vector bundle and that $(T(\xi), T(X))$ and $(T(\eta), T(Y))$ are linear sections of $T D$ to the reader.

The warp w $(\xi, \eta) \in \Gamma C$ of $(\xi, X),(\eta, Y)$ is given as usual by (8). We calculate the warp of the tangent grid. From the definition of a warp, for $x \in T_{m} M$,

$$
(T(\xi) \circ T(Y))(x) \frac{-}{T A}(T(\eta) \circ T(X))(x)=T\left(0^{D} \circ X\right)(x) \underset{T B}{+} \mathrm{w}(T(\xi), T(\eta))(x) .
$$

Write $x=\left.\frac{d}{d t} m_{t}\right|_{t=0}$, for $m_{t}$ a curve in $M$ with tangent vector $x$ at $t=0$. Then, for $F \in C^{\infty}(D)$,

$$
\begin{aligned}
& \left((T(\xi) \circ T(Y)) \frac{-}{T A}(T(\eta) \circ T(X))\right)(x)(F) \\
& =\left.\frac{d}{d t} F\left((\xi \circ Y)\left(m_{t}\right) \frac{-}{A}(\eta \circ X)\left(m_{t}\right)\right)\right|_{t=0} \\
& =\frac{d}{d t} F\left(\left.0_{X\left(m_{t}\right)}^{D} \underset{B}{\left.+\mathrm{w}(\xi, \eta)\left(m_{t}\right)\right)}\right|_{t=0}\right. \\
& =\left.\frac{d}{d t} F\left(\left(0^{D} \circ X\right)\left(m_{t}\right)+\underset{B}{\mathrm{w}}(\xi, \eta)\left(m_{t}\right)\right)\right|_{t=0} \\
& =\left(T\left(0^{D} \circ X\right)(x) \underset{T B}{+} T(\mathrm{w}(\xi, \eta))(x)\right)(F) .
\end{aligned}
$$

Here we used the formula

$$
\left(T(\xi)\left(\Phi_{1}\right) \underset{T A}{+} T(\xi)\left(\Phi_{2}\right)\right)(F)=\left.\frac{d}{d t} F\left(\xi \circ \varphi_{t}^{1}+\underset{A}{\xi} \circ \varphi_{t}^{2}\right)\right|_{t=0}
$$

for the addition in $T D \rightarrow T A$, and the corresponding formula for scalar multiplication. In (69), where $F \in C^{\infty}(D)$, the $\Phi_{i}$ are elements of $T B$ and the $\varphi_{t}^{i}$ are curves in $B$ with

$$
\Phi_{i}=\left.\frac{d}{d t} \varphi_{t}^{i}\right|_{t=0}, \quad i=1,2
$$


Given $T\left(q_{B}\right)\left(\Phi_{1}\right)=T\left(q_{B}\right)\left(\Phi_{2}\right)$ we can arrange that $q_{B}\left(\varphi_{t}^{1}\right)=q_{B}\left(\varphi_{t}^{2}\right)$ for $t$ near zero. By uniqueness of the core element, it follows from (68) that

$$
\mathrm{w}(T(\xi), T(\eta))(x)=T(\mathrm{w}(\xi, \eta))(x) .
$$

Applying Propositition 4 to the grid on the Up face of (65) it follows that

$$
\mathrm{w}_{\mathrm{up}}=T\left(\nabla_{X} \mu\right) \in \Gamma_{T M}(T A) .
$$

To calculate the warp of the Back face, we need to use the canonical involution $J_{A}$. The warp of the Back face is given by

$$
T^{2}(\mu)(\widetilde{Z}(X(m))) \underset{p_{T A}}{-Z^{H}}{ }^{A}(T(\mu)(X(m))) .
$$

The outlines of the two elements are
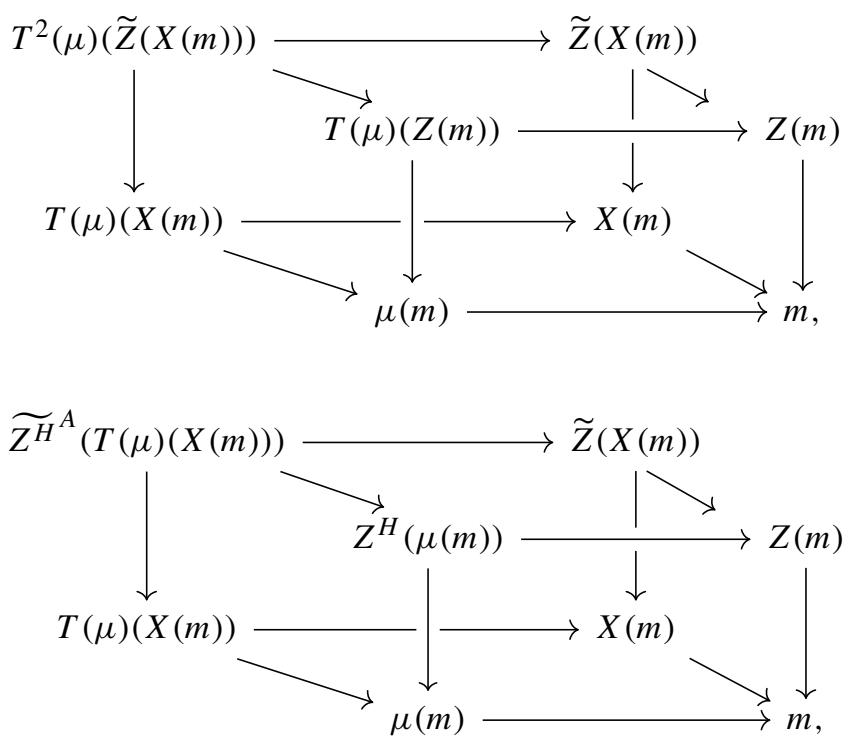

(compare with the general triple outlines of the elements $Y Z X$ and ZYX, of Sect. 4.1).

Writing the complete lifts as $\widetilde{Z^{H}}{ }^{A}=J_{A} \circ T\left(Z^{H}\right)$ and $\widetilde{Z}=J_{M} \circ T(Z)$, and using the naturality of $J$-maps (Lemma 2), we have that

$$
\begin{aligned}
& T^{2}(\mu)(\widetilde{Z}(X(m))) \underset{p_{T A}}{\widetilde{Z}^{H}}(T(\mu)(X(m))) \\
& \quad=T^{2}(\mu)\left(J_{M}(T(Z)(X(m)))\right) \frac{-}{p_{T A}} J_{A}\left(T\left(Z^{H}\right)(T(\mu)(X(m)))\right) \\
& \quad=J_{A}\left(T^{2}(\mu)(T(Z)(X(m)))\right) \frac{-}{p_{T A}} J_{A}\left(T\left(Z^{H}\right)(T(\mu)(X(m)))\right) .
\end{aligned}
$$


Since $J_{A}$ interchanges the structures $p_{T A}$ and $T\left(p_{A}\right)$, we can rewrite the last expression in (70) as

$$
J_{A}\left(T^{2}(\mu)(T(Z)(X(m))) \underset{T\left(p_{A}\right)}{-} T\left(Z^{H}\right)(T(\mu)(X(m)))\right) .
$$

Focus on $T^{2}(\mu)(T(Z)(X(m))) \underset{T\left(p_{A}\right)}{-} T\left(Z^{H}\right)(T(\mu)(X(m)))$. We can rewrite this as

$$
T(T(\mu) \circ Z)(X(m)) \underset{T\left(p_{A}\right)}{-} T\left(Z^{H} \circ \mu\right)(X(m)) .
$$

At this point, we apply Proposition 4 to the following grid,

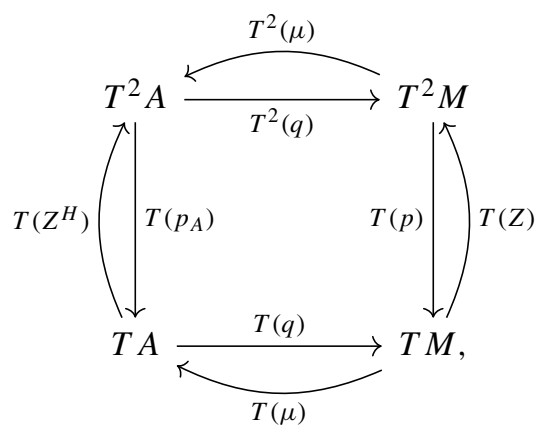

which is precisely the tangent of the grid (9). The warp of the latter is $\nabla_{Z} \mu$. Therefore, by Proposition 4, the warp of the tangent of (9) is given, for any $x \in T_{m} M$, by

$$
\begin{gathered}
\left(T^{2}(\mu) \circ T(Z)\right)(x) \underset{T\left(p_{A}\right)}{-}\left(T\left(Z^{H}\right) \circ T(\mu)\right)(x) \\
={\overline{T\left(\nabla_{Z} \mu\right)(x)}}^{+\underset{T^{2}(q)}{+} T\left(\tilde{0}^{T A}\right)(T(\mu)(x)) .}
\end{gathered}
$$

Here we have denoted by $\tilde{0}^{T A}$ the zero section of $T A \stackrel{p_{A}}{\longrightarrow} A$. For $x=X(m)$, the right-hand side of (72) is equal to (71). Therefore, (71) is equal to

$$
{\overline{T\left(\nabla_{Z} \mu\right)(X(m))}}^{U} \underset{T^{2}(q)}{+} T\left(\tilde{0}^{T A}\right)(T(\mu)(X(m))) .
$$

We return now to our calculation of (70). Applying $J_{A}$ to (73), we have that (70) is

$$
J_{A}\left({\overline{T\left(\nabla_{Z} \mu\right)(X(m))}}^{U}\right) \underset{T^{2}(q)}{+} J_{A}\left(T\left(\tilde{0}^{T A}\right)(T(\mu)(X(m)))\right) .
$$

The addition over $T^{2}(q)$ does not change under $J_{A}$, by Lemma 3. From (63) we have 


$$
\begin{aligned}
& J_{A}\left(\overline{T\left(\nabla_{Z} \mu\right)(X(m))}\right)^{U} \underset{T^{2}(q)}{+} J_{A}\left(T\left(\tilde{0}^{T A}\right)(T(\mu)(X(m)))\right) \\
& \quad={\overline{T\left(\nabla_{Z} \mu\right)(X(m))}}^{B} \underset{T^{2}(q)}{+} 0_{T(\mu)(X(m))}^{T^{2} A} .
\end{aligned}
$$

This completes the calculation of the warp of the Back face; taking into consideration the orientation of the Back face, the warp is $\mathrm{w}_{\mathrm{back}}=-T\left(\nabla_{Z} \mu\right) \in \Gamma_{T M} T A$.

Finally, the warp of the Left face is the crux of this example. The Left face is the double tangent vector bundle $T^{2} A$ for the manifold $A$. We therefore apply (5). Taking into account the orientation of the Left face, we have

$$
T\left(X^{H}\right) \circ Z^{H}-\widetilde{Z^{H}}{ }^{A} \circ X^{H} \triangleright\left[Z^{H}, X^{H}\right] .
$$

What is $\left[Z^{H}, X^{H}\right]$ ? When a connection in a vector bundle is formulated in terms of a lifting of vector fields from the base to the total space, as in Example 1, the difference $\left[Z^{H}, X^{H}\right]-[Z, X]^{H}$ is one formulation of the curvature of the connection.

Using the warp theorem, we now show that the warp $\left[Z^{H}, X^{H}\right]$, combined with the other warp terms from the grid (65), yields the standard formula for curvature in terms of covariant derivatives. For the full statement see Theorem 2.

To begin, note that both $\left[Z^{H}, X^{H}\right]$ and $[Z, X]^{H}$ project to $[Z, X]$ and therefore their difference is a linear and vertical vector field on $A$.

At this point we take a closer look at linear sections $\left(\eta, 0^{B}\right)$, where $\eta \in \Gamma_{A} D$ and $0^{B} \in \Gamma B$, of a general double vector bundle $D$.

Lemma 4 Let $\varphi: A \rightarrow C$ be a vector bundle map over $M$. Define $\varphi^{\zeta}: A \rightarrow D$ by

$$
\varphi^{\zeta}(a)=\varphi(a)+{ }_{B} 0_{a}^{D} .
$$

Then $\left(\varphi^{\zeta}, 0^{B}\right)$ is a linear section. Every linear section $\left(\eta, 0^{B}\right)$ is of this form for a unique $\varphi: A \rightarrow C$.

We call $\left(\varphi^{\zeta}, 0^{B}\right)$ the bolt of $\varphi$; in [7] they are called core-linear sections.

Proof That $\left(\varphi^{4}, 0^{B}\right)$ is a linear section follows immediately from the definition of $\varphi^{4}$. Take $a_{1}, a_{2} \in A_{m}$. Then

$$
\begin{aligned}
& \varphi^{4}\left(a_{1}+a_{2}\right)=\varphi\left(a_{1}+a_{2}\right)+{ }_{B} 0_{a_{1}+a_{2}}^{D}=\left(\varphi\left(a_{1}\right)+\underset{B}{ } \varphi\left(a_{2}\right)\right)+{ }_{B}\left(0_{a_{1}}^{D}+{ }_{B} 0_{a_{2}}^{D}\right) \\
& =\left(\varphi\left(a_{1}\right)+{ }_{B} 0_{a_{1}}^{D}\right){ }_{B}+\left(\varphi\left(a_{2}\right)+{ }_{B} 0_{a_{2}}^{D}\right)=\varphi^{\zeta}\left(a_{1}\right)+{ }_{B} \varphi^{\zeta}\left(a_{2}\right),
\end{aligned}
$$

and similarly for the scalar multiplication.

Suppose now that $\left(\eta, 0^{B}\right)$ is a linear section. For $a \in A_{m}, \eta(a)$ has the following outline:

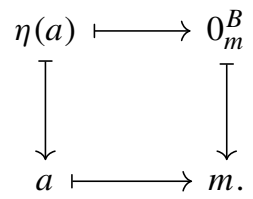


Therefore, we can write $\eta(a)=c+\underset{B}{0} 0_{a}^{D}$, for a unique $c \in C_{m}$. Define $\varphi(a)=c$. Then

$$
\eta(a)=\varphi(a)+0_{B}^{D} .
$$

To show that $\varphi$ is a vector bundle morphism, take $a_{1}, a_{2} \in A_{m}$. Then

$$
\varphi\left(a_{1}+a_{2}\right)=\varphi\left(a_{1}\right)+\varphi\left(a_{2}\right)
$$

follows immediately from the linearity of $\eta$. And of course, similarly for the scalar multiplication. Finally, we easily see that $\varphi^{\zeta}=\eta$.

The following notation prepares the way for Theorem 2 .

Definition 7 With the above notation, write $R: A \rightarrow A$ for the vector bundle map such that

$$
[Z, X]^{H}-\left[Z^{H}, X^{H}\right]=R^{\zeta} \text {. }
$$

It will take us until the end of the section to show that this definition leads to the usual concept of curvature.

\subsection{The three ultrawarps}

The second and final step in applying the warp theorem to the grid (65) on $T^{2} A$ consists of calculating the ultrawarps of the induced grids on the core double vector bundles of $T^{2} A$.

In Table 2 we present the induced grids and the ultrawarps, and we explain the calculations in the remainder of the section.

The ultrawarps for the (B-F) and the (U-D) core double vector bundles (first and third row of Table 2) follow directly from Example 1, taking of course into account the orientation of the core double vector bundles.

What requires some work is the ultrawarp of the induced grid on the (L-R) core double vector bundle (second row of Table 2). Taking into account the orientation of the $(\mathrm{L}-\mathrm{R})$ core double vector bundle, this ultrawarp is defined by

$$
\left[Z^{H}, X^{H}\right] \circ \mu-T(\mu) \circ[Z, X] .
$$

This grid is of a new type; we encounter it here for the first time. Using Definition 7, we rearrange it as

$$
\left[Z^{H}, X^{H}\right]=[Z, X]^{H}-R^{\zeta} \text {. }
$$

We need a small parenthesis on bolts and warps. It is easily seen that the warp of a horizontal linear section $(\xi, X)$ and a bolt $\left(\varphi^{\zeta}, 0^{B}\right)$ is

$$
\mathrm{w}\left(\xi, \varphi^{\zeta}\right)=-\varphi \circ X .
$$

In addition, we have the following proposition. 
Table 2 Induced grids and ultrawarps in $T^{2} A$

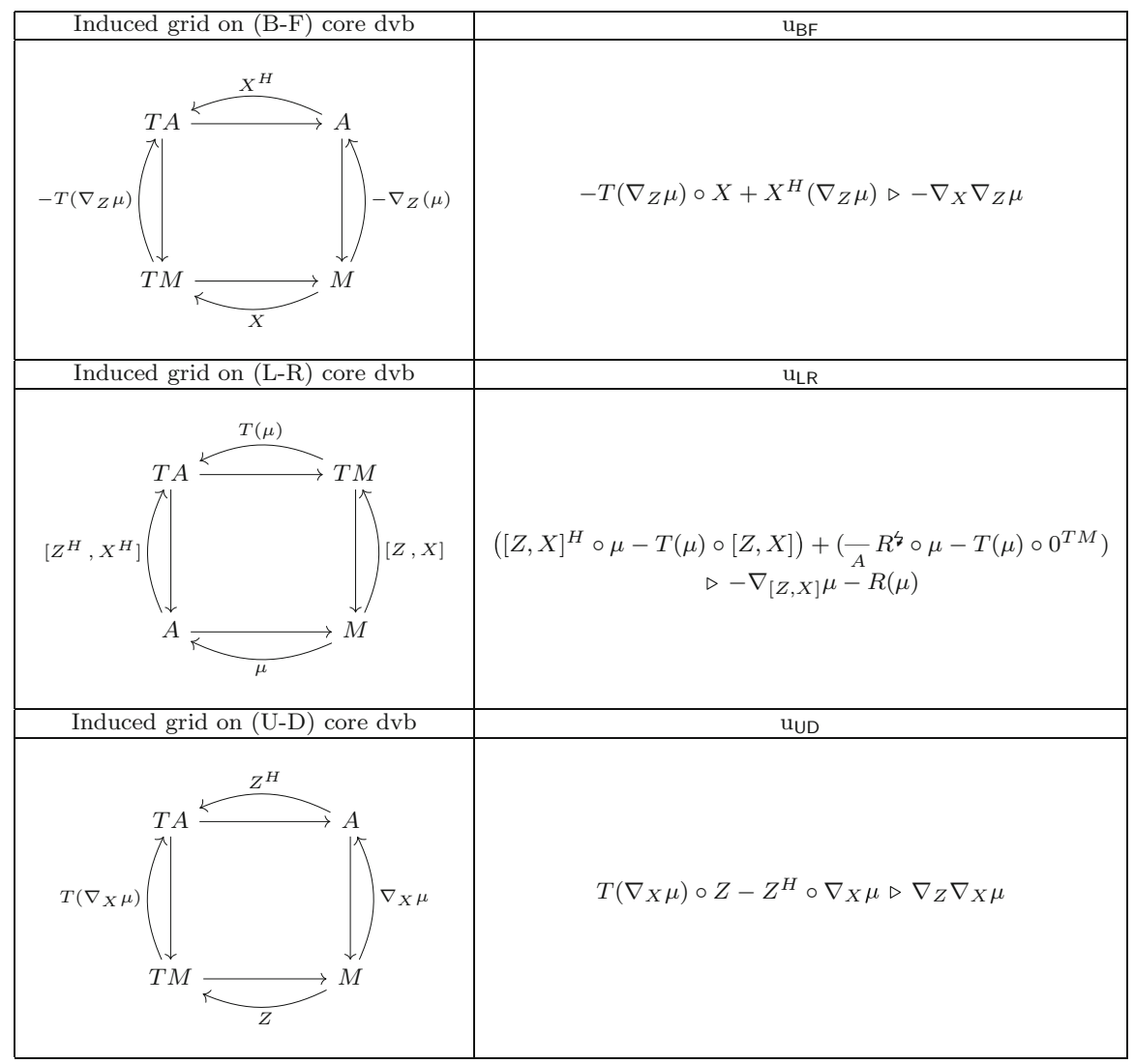

Proposition 5 Given two grids $(\xi, X),(\eta, Y)$, and $(\xi, X),\left(\varphi^{\zeta}, 0^{B}\right)$ on a double vector bundle D,
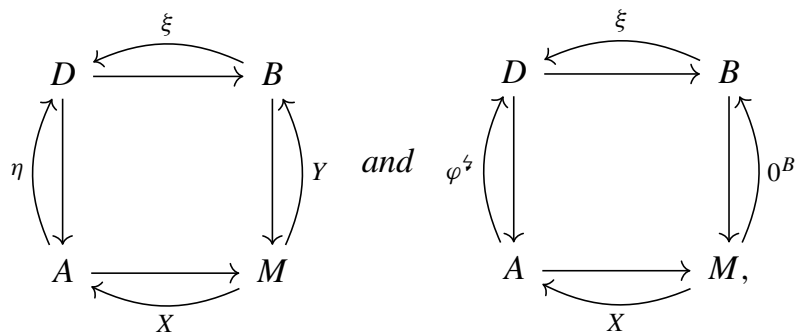

with warps $\mathrm{w}(\xi, \eta)$ and $\mathrm{w}\left(\xi, \varphi^{\zeta}\right)$, then

$$
\mathrm{w}\left(\xi, \eta \underset{A}{+} \varphi^{\xi}\right)=\mathrm{w}(\xi, \eta)+\mathrm{w}\left(\xi, \varphi^{\xi}\right) .
$$


We leave the proof to the reader.

Using Proposition 5, we can rewrite the induced grid on the (L-R) core double vector bundle as the sum of
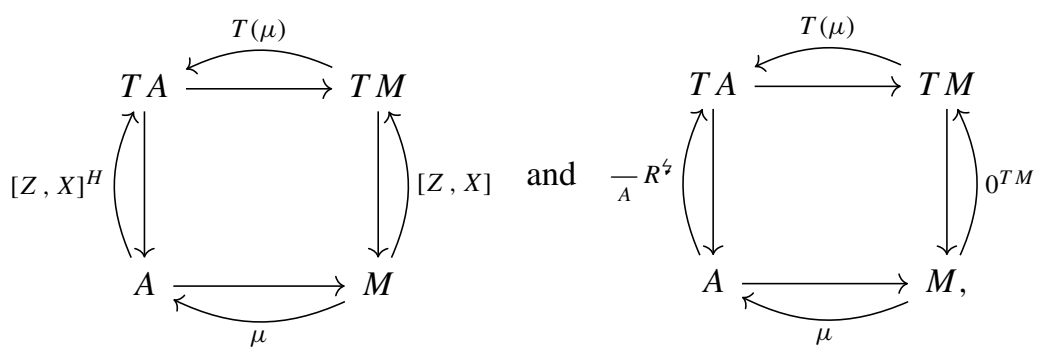

so (75) is now, from Proposition 5,

$$
\left([Z, X]^{H} \circ \mu-T(\mu) \circ[Z, X]\right)+\left(\left(\frac{-}{A} R^{\zeta}\right) \circ \mu-T(\mu) \circ 0^{T M}\right) .
$$

The warp of the first grid of (77) is $-\nabla_{[Z, X]} \mu$ (see Example 1). The warp of the second grid of (77), using (76), is $-R(\mu)$. So in total, the warp of the induced grid on the (L-R) core double vector bundle is

$$
-\nabla_{[Z, X]} \mu-R(\mu)
$$

The warp theorem now gives us that

$$
-\nabla_{X} \nabla_{Z} \mu-\nabla_{[Z, X]} \mu-R(\mu)+\nabla_{Z} \nabla_{X} \mu=0 .
$$

Rearranging, we have the standard formula for curvature in terms of covariant derivatives.

The use of covariant derivatives and the use of horizontal lifts are the two usual methods for working globally with connections in vector bundles. The covariant derivative formulation was a natural development from the special cases of surface theory and Riemannian geometry. It is our impression that the use of horizontal lifts emerged from comparison with the connection theory of principal bundles.

As noted on page 7, Kobayashi and Nomizu [11] gave two equivalent global formulations of the concept of connection in a principal bundle $P(M, G)$ : as a suitable $\mathfrak{g}$-valued 1-form on $P$ and as an invariant horizontal distribution $\mathscr{H}$ on $P$. They concentrated on the first of these, and its local version. It was noticed early on that a connection was flat if and only if the corresponding $\mathscr{H}$ was involutive, and that the choice of $\mathscr{H}$ was equivalent to a lifting of vector fields on $M$ to invariant vector fields on $P$ : putting these together led to a definition of curvature by precisely the formula (74) above. It was then easy to transfer this principal bundle formulation to vector bundles.

Both Dieudonné [4, 17.20] and Greub et al. [10, VII,§4] obtained (78) by iterating the covariant exterior derivative. What we have done is obtain (78) independently of 
earlier treatments, as a consequence of the warp theorem; if we start with the concept of a connection $\nabla$, and apply the warp theorem to the grid (65) on $T^{2} A$, we obtain (78) for $R(\mu)$. In other words, we have the following theorem.

Theorem 2 Given a connection $\nabla$ in $(A, q, M)$ and the equivalent horizontal lifting $Z \mapsto Z^{H}$ as defined in (9), the vector bundle map $R: A \rightarrow A$ for which the bolt $R^{\zeta}$ is given by (74) is the curvature $R_{\nabla}(Z, X)$ as defined in (78).

\section{The triple tangent bundle $T^{3} M$ and the Jacobi identity}

In this section we consider the triple tangent bundle $T^{3} M$ of a manifold $M$ and construct a grid on it, for which the Jacobi identity emerges as a consequence of the warp theorem. A version of this approach was given by Mackenzie [18]. We present here a clearer and more detailed calculation.

Take $E$ to be $T^{3} M$, the triple tangent bundle. This is a special case of $T^{2} A$, for $A=T M$ :

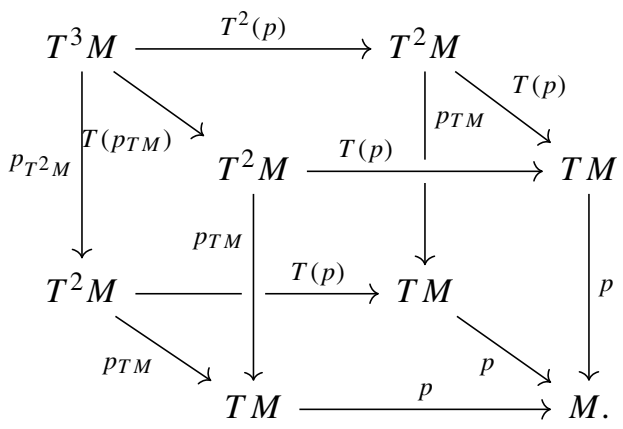

The three lower faces are copies of $T^{2} M$. The Left face is the double tangent bundle of the manifold $T M$. The Back face is not a double tangent bundle; it is the tangent double vector bundle of $T^{2} M \stackrel{T(p)}{\longrightarrow} T M$. The Up face is obtained by applying the tangent functor to $T^{2} M$.

Starting with three vector fields $X, Y$, and $Z$, each a section of one of the three copies of $T M$, one can build a grid on $T^{3} M$ as follows; see (79) below.

- The front-back linear double section $(T(\tilde{X}) ; \tilde{X}, T(X) ; X)$. Take the complete lift of $X$ across the Down face, and apply the tangent functor to the linear section $(\widetilde{X}, X)$.

- The right-left linear double section $\left(T^{2}(Y) ; T(Y), T(Y) ; Y\right)$. Apply the tangent functor to $Y$ and then to $T(Y)$.

- The down-up linear double section $(\widetilde{Z} ; \widetilde{Z}, \widetilde{Z} ; Z)$. Take the complete lift of $Z$ across the Front face, and the complete lift of this across the Left face. Likewise take the complete lift of $Z$ across the Right face. One does need to check that $(\widetilde{\widetilde{Z}}, \widetilde{Z})$ is indeed a linear section of the Back face. 
The diagram in (79) shows the entire grid.

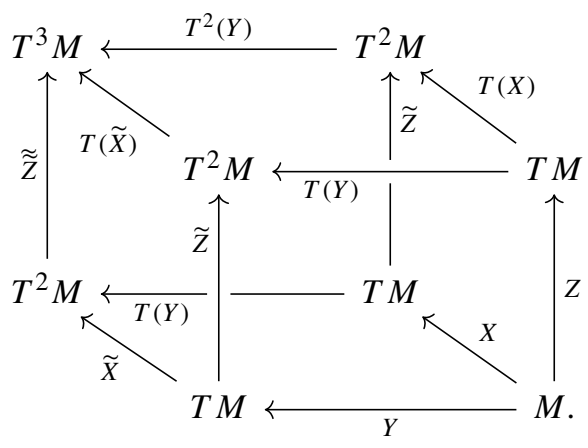

We now calculate the three ultrawarps defined by this grid. To do this, we calculate the core morphisms of the three linear double sections, and the warps of the six faces.

First, the core morphisms. These follow in an analogous way as in the example of $T^{2} A$,

- The core morphism of $(T(\tilde{X}) ; \tilde{X}, T(X) ; X)$ is $(\tilde{X}, X)$.

- The core morphism of $\left(T^{2}(Y) ; T(Y), T(Y) ; Y\right)$ is $(T(Y), Y)$.

- The core morphism of $(\widetilde{\widetilde{Z}} ; \widetilde{Z}, \widetilde{Z} ; Z)$ is $(\widetilde{Z}, Z)$.

To calculate the warps of the six faces, we take into consideration the orientation of the faces of a triple vector bundle. For the lower faces, by (5):

- For the Front face: $\quad \widetilde{Z}(Y)-T(Y)(Z) \triangleright[Y, Z]$.

- For the Right face: $\quad T(X)(Z)-\widetilde{Z}(X) \triangleright[Z, X]$.

- For the Down face: $\quad T(Y)(X)-\widetilde{X}(Y) \triangleright[X, Y]$.

We now calculate the warps of the upper faces.

Back face. The warp of the Back face, for $x \in T M$, is given by

$$
\widetilde{Z} \circ T(Y)(x) \underset{T^{2}(p)}{-} T^{2}(Y) \circ \widetilde{Z}(x)=\mathrm{w}_{\mathrm{back}}(x) \underset{p_{T^{2} M}^{+}}{+} \hat{0} \widetilde{Z}(x) .
$$

As noted, the Back face is the tangent double vector bundle of $T^{2} M \stackrel{T(p)}{\longrightarrow} T M$. Apply $T(J)$ to it, the tangent of the canonical involution $J: T^{2} M \rightarrow T^{2} M$. The resulting double vector bundle is now the double tangent bundle of $T M$. In fact, $T(J)$ is a triple vector bundle morphism, and maps the Back face of $T^{3} M$ to the double tangent bundle 
of $T M$ as shown in (81).

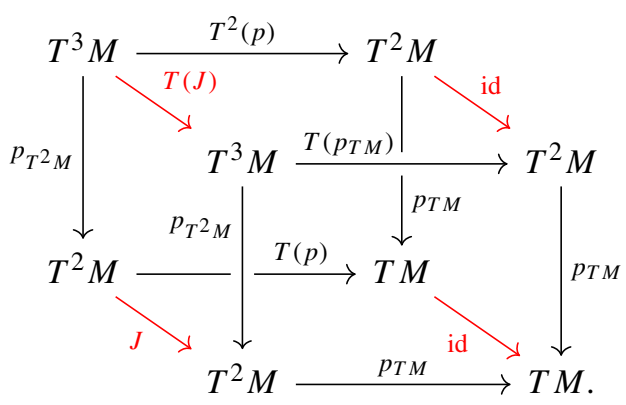

Also, the core morphism of (81) is $(J$, id). Hence, applying $T(J)$ to $(80)$,

$$
T(J)\left(\widetilde{\widetilde{Z}} \circ T(Y)(x) \underset{T^{2}(p)}{-} T^{2}(Y) \circ \widetilde{Z}(x)\right)=J\left(\mathrm{w}_{\text {back }}(x)\right) \underset{p_{T^{2} M}^{+}}{+} \hat{0}_{\widetilde{Z}(x)} .
$$

Note that $T(J)$ changes the vector bundle structure over which the subtraction of the

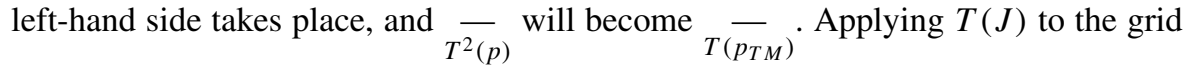
of the Back face yields the following grid on the double tangent bundle of $T M$.

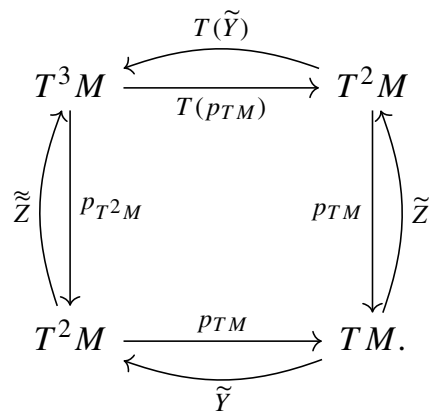

Therefore, expanding the left-hand side of (82),

$$
\begin{aligned}
& T(J)\left(\widetilde{\widetilde{Z}} \circ T(Y)(x) \underset{T^{2}(p)}{-} T^{2}(Y) \circ \widetilde{Z}(x)\right) \\
& =T(J)((\widetilde{\widetilde{Z}} \circ T(Y))(x))_{T\left(\overline{p_{T} M}\right)} T(J)\left(\left(T^{2}(Y) \circ \widetilde{Z}\right)(x)\right) \\
& =(\widetilde{Z} \circ \widetilde{Y})(x) \underset{T\left(p_{T M}\right)}{-}(T(\tilde{Y}) \circ \widetilde{Z})(x) \\
& \stackrel{(5)}{=}-[\widetilde{Z}, \widetilde{Y}](x) \underset{p_{T^{2} M}}{+} \hat{0}_{\widetilde{Z}(x)}=\widetilde{[Y, Z]}(x) \underset{p_{T^{2} M}}{+} \hat{0} \widetilde{Z}(x) .
\end{aligned}
$$


Substituting this into (82),

$$
\widetilde{[Y, Z]}(x) \underset{p_{T^{2} M}}{+} \hat{0} \widetilde{Z}(x)=J\left(\mathrm{w}_{\mathrm{back}}(x)\right) \underset{p_{T^{2} M}}{+} \hat{0}_{\widetilde{Z}(x)},
$$

and using that $J^{2}=\mathrm{id}$, we obtain

$$
\mathrm{w}_{\mathrm{back}}=T([Y, Z]) .
$$

Left face. The Left face is the double tangent bundle of $T M$, so we simply apply (5) to the grid $(T(\widetilde{X}), \widetilde{X}),(\widetilde{\widetilde{Z}}, \widetilde{Z})$, and obtain

$$
T(\tilde{X}) \circ \widetilde{Z}-\widetilde{\widetilde{Z}} \circ \widetilde{X} \triangleright[\widetilde{Z}, \widetilde{X}]=\widetilde{[Z, X]},
$$

so $\mathrm{w}_{\text {left }}=\widetilde{[Z, X]}$.

Up face. For the Up face, using Proposition $4, \mathrm{w}_{\mathrm{up}}=T([X, Y])$ follows directly.

The three ultrawarps. The three core double vector bundles are all copies of $T^{2} M$, and their ultracore is $T M \rightarrow M$.

The three core double vector bundles in the usual order (B-F), (L-R), and (U-D), with the induced grids from the original grid on $T^{3} M$,

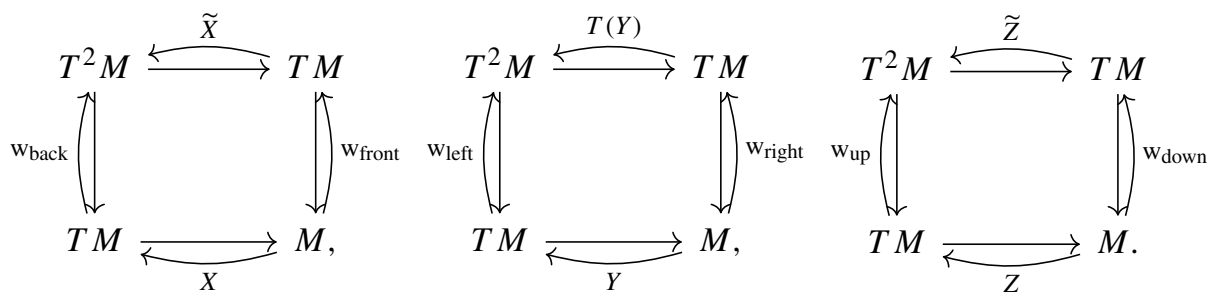

Finally, by (5), the ultracore elements are

$$
\begin{aligned}
& \mathrm{w}_{\text {back }} \circ X-\tilde{X} \circ \mathrm{w}_{\text {front }}=T([Y, Z]) \circ X-\tilde{X} \circ[Y, Z] \triangleright[X,[Y, Z]], \\
& \mathrm{w}_{\text {left }} \circ Y-T(Y) \circ \mathrm{w}_{\text {right }}=\widehat{[Z, X]} \circ Y-T(Y) \circ[Z, X] \triangleright[Y,[Z, X]] \text {, } \\
& \mathrm{w}_{\mathrm{up}} \circ Z-\widetilde{Z} \circ \mathrm{w}_{\text {down }}=T([X, Y]) \circ Z-\widetilde{Z} \circ[X, Y] \triangleright[Z,[X, Y]] \text {. }
\end{aligned}
$$

We see that in this way we have formulated the three terms of the Jacobi identity. And applying the warp theorem, we obtain a diagrammatic proof of the Jacobi identity.

\section{Concluding remarks}

The statement of the warp theorem is easy to describe and needs only minimal notation (in fact it reminds us of the "boundary of the boundary is zero" for cochains), but to 
prove it globally we have had to introduce a considerable arsenal of background techniques, and make a number of intricate calculations. This is an instance of the great difference between geometric intuition and rigor.

It is natural to consider whether the horizontal lifting process of Sect. 5 and the complete lifting of Sect. 6 can be unified by a general process of lifting. This seems to us unlikely, since complete and horizontal lifts differ in many respects. The warp theorem applies to both cases and can be regarded as providing as much unification as is possible.

In a future paper we will consider in detail the application of the warp theorem to triple vector bundles with compatible bracket structures.

Acknowledgements We are very grateful to Yvette Kosmann-Schwarzbach, Ted Voronov and Ping Xu for valuable comments at various stages in the preparation of this paper. We also send our best thanks to Fani Petalidou for thorough readings of earlier versions of the paper and for catching many slips. We especially appreciate the comments of the referee, which have clarified and enhanced our own understanding of the results.

Open Access This article is distributed under the terms of the Creative Commons Attribution 4.0 International License (http://creativecommons.org/licenses/by/4.0/), which permits unrestricted use, distribution, and reproduction in any medium, provided you give appropriate credit to the original author(s) and the source, provide a link to the Creative Commons license, and indicate if changes were made.

\section{References}

1. Abraham, R., Marsden, J.E., Ratiu, T.: Manifolds, Tensor Analysis, and Applications, Applied Mathematical Sciences, vol. 75, 2nd edn. Springer, New York (1988)

2. Besse, A.L.: Manifolds all of whose geodesics are closed, Ergebnisse der Mathematik und ihrer Grenzgebiete [Results in Mathematics and Related Areas], vol. 93. Springer, Berlin (1978). With appendices by D. B. A. Epstein, J.-P. Bourguignon, L. Bérard-Bergery, M. Berger and J. L. Kazdan

3. Courant, T.: Tangent Dirac structures. J. Phys. A 23(22), 5153-5168 (1990). http://stacks.iop.org/ $0305-4470 / 23 / 5153$

4. Dieudonné, J.: Treatise on analysis. Vol. III. Academic Press, New York (1972). Translated from the French by I. G. MacDonald, Pure and Applied Mathematics, Vol. 10-III

5. Flari, M. K.: Triple vector bundles in Differential Geometry, PhD thesis, University of Sheffield (2018)

6. Grabowski, J., Rotkiewicz, M.: Higher vector bundles and multi-graded symplectic manifolds. J. Geom. Phys. 59(9), 1285-1305 (2009)

7. Gracia-Saz, A., Jotz Lean, M., Mackenzie, K.C.H., Mehta, R.A.: Double Lie algebroids and representations up to homotopy. J. Homotopy Relat. Struct. 13(2), 287-319 (2018). https://doi.org/10.1007/ s40062-017-0183-1

8. Gracia-Saz, A., Mackenzie, K.C.H.: Duality functors for $n$-fold vector bundles. arXiv:1209.0027

9. Gracia-Saz, A., Mackenzie, K.C.H.: Duality functors for triple vector bundles. Lett. Math. Phys. 90(13), 175-200 (2009). https://doi.org/10.1007/s11005-009-0346-z

10. Greub, W., Halperin, S., Vanstone, R.: Connections, Curvature, and Cohomology, Vol. II: Lie Groups, Principal Bundles, and Characteristic Classes. Academic Press, New York (1973)

11. Kobayashi, S., Nomizu, K.: Foundations of Differential Geometry. Vol. I Interscience Publishers. Wiley, New York (1963)

12. Koszul, J.L.: Lectures on fibre bundles and differential geometry. Notes by S. Ramanan. Tata Institute of Fundamental Research Lectures on Mathematics, No. 20. Tata Institute of Fundamental Research, Bombay (1965)

13. Li-Bland, D., Ševera, P.: Quasi-Hamiltonian groupoids and multiplicative Manin pairs. Int. Math. Res. Not. IMRN 10, 2295-2350 (2011). https://doi.org/10.1093/imrn/rnq170

14. Mackenzie, K.C.H.: Double Lie algebroids and second-order geometry. I. Adv. Math. 94(2), 180-239 (1992). https://doi.org/10.1016/0001-8708(92)90036-K 
15. Mackenzie, K.C.H.: Duality and triple structures. In: The breadth of symplectic and Poisson geometry, Progr. Math., vol. 232, pp. 455-481. Birkhäuser, Boston, MA (2005)

16. Mackenzie, K.C.H.: General theory of Lie groupoids and Lie algebroids, London Mathematical Society Lecture Note Series, vol. 213. Cambridge University Press, Cambridge (2005)

17. Mackenzie, K.C.H.: Ehresmann doubles and Drinfel'd doubles for Lie algebroids and Lie bialgebroids. J. Reine Angew. Math. 658, 193-245 (2011)

18. Mackenzie, K.: Proving the Jacobi identity the hard way. In: Geometric methods in physics, Trends Math., pp. 357-366. Birkhäuser, Basel (2013)

19. Mackenzie, K.C.H., Xu, P.: Lie bialgebroids and Poisson groupoids. Duke Math. J. 73(2), 415-452 (1994). https://doi.org/10.1215/S0012-7094-94-07318-3

20. Misner, C.W., Thorne, K.S., Wheeler, J.A.: Gravitation. W. H. Freeman and Co., San Francisco (1973)

21. Pradines, J.: Fibrés vectoriels doubles et calcul des jets non holonomes, Esquisses Mathématiques [Mathematical Sketches], vol. 29. Université d'Amiens, U.E.R. de Mathématiques, Amiens (1977) 